TRANSACTIONS OF THE

AMER ICAN MATHEMATICAL SOCIETY

Volume 169 , July 1972

\title{
HARMONIC ANALYSIS ON $F$-SPACES WITH A BASIS
}

BY

\section{J. KUELBS( $\left.{ }^{1}\right)$ AND V. MANDREKAR( 2 )}

ABSTRACT. We establish Bochner's theorem and the Levy continuity theorem in the case that the underlying space is a real $F$-space with a basis, and then examine the infinitely divisible probability measures on a class of such spaces.

1. Introduction. We generalize Bochner's theorem and the Levy continuity theorem of Fourier analysis to the case that the underlying space $E$ is a real $F$ space with basis and then study the infinitely divisible measures on such spaces. This extends the results of [2] which handles the situation where $E$ is a Hilbert space and of [3] where $E$ was an $l_{p}$ space, $0<p<\infty$. It is also related to the work of V. Sazonov ([6], [7]), Yu. V. Prokhorov ([5], [6]), and to the study of the infinitely divisible measures on a separable Hilbert space which is due to S.R.S. Varadhan in [11].

We first establish some terminology in $\$ 2$. In $\$ 3$ we give Bochner's theorem (Theorem 3.1) and in Corollary 3.1 we link Theorem 3.1 to the results of [2] and [3]. $\$ 4$ is devoted to the continuity theorem. These results are of some interest in themselves but it is their application to Gaussian measures and other infinite ly divisible laws which is of most interest to us. In fact, the proofs of Theorem 3.1 and Theorem 4.1 are much the same as those given in [3] when $E=l_{p}(2 \leq p<\infty)$ so we do not provide the details here.

In $\$ 5$ we give some applications to Gaussian measures. In particular, Theorem 5.1 characterizes the Fourier transform of a Gaussian measure, Theorem 5.2 gives necessary and sufficient conditions in terms of the Fourier transforms for a family of Gaussian measures to be conditionally compact, and Theorem 5.3 deals with the convergence of Gaussian measures on $E$ provided, in each case, $E$ has what we call Property B. Corollaries 5.2 and 5.3 then relate these results to the case $E=l_{p}, 2 \leq p<\infty$. In fact, Corollary 5.3 was previously known to N. N. Vakhania ([8], [9]). Finally, in $\$ 6$ we characterize a large class of spaces whose quasinorm is accessible in both directions as Orlicz spaces. This result is found in

Received by the editors May 17, 1971.

AMS 1969 subject classifications. Primary 6008, 6030; Secondary 2846.

Key words and phrases. Bochner theorem, Levy continuity theorem, Gaussian measures, infinitely divisible probability measures, central limit theorem, Orlicz space, uniformity.

(1) Supported in part by NSF Grant GP-9372.

(2) Supported in part by NSF Grant GP-11626. 
Theorem 6.3 and Corollary 6.2 again relates to the $l_{p}$ spaces, $2 \leq p<\infty$. In Corollary 6.3 we also have a similar characterization of those spaces with Property $B$.

In the last sections of the paper we study the infinitely divisible laws on these Orlicz spaces. In particular, $\$ 7$ is devoted to the representation theorem of the Fourier transform of an infinitely divisible law, $\$ 8$ centers on the limiting be havior of sums of small independent random variables with values in the Orlicz spaces, and in $\$ 9$ we apply the results of $\$ 8$ to obtain a central limit theorem.

2. Terminology and preliminaries. Throughout the paper, $E$ will denote a real $F$-space with basis $\left\{b_{n}\right\}$. Here, as is usual, an $F$-space is a topological vector space whose topology is given by an invariant metric, i.e. $d(x, y)=d(x-y, 0)$, which is also complete in this metric. We will let $\|x\|=d(x, 0)$. Then $\|\cdot\|$ has all the properties of a norm, except possibly homogeneity, and since $E$ is a topological vector space it does satisfy the property that $\lim _{n}\left\|a_{n} x_{n}-a x\right\|=0$ whenever $\left\{a_{n}\right\}$ is a sequence of reals converging to $a$ and $\left\{x_{n}\right\}$ is a sequence in $E$ converging to $x$. In this case, we will refer to $\|\cdot\|$ as a quasi-norm so as to distinguish it from a norm.

Since $E$ has a basis, for each $x \in E$ there is a unique sequence of real numbers $\left\{\beta_{n}\right\}$ such that $\lim _{k}\left\|x-\sum_{n=1}^{k} \beta_{n} b_{n}\right\|=0$, and it is easy to see that $E$ is separable. We will write the expansion of $x$ as $\sum_{n=1}^{\infty} \beta_{n}(x) b_{n}$ and this emphasizes that the coefficients generate coordinate functionals on $E$. It is clear that these coordinate functionals are linear and it is well known that they are continuous as well. Further, it is possible to assume without loss of generality (and we do) that $\|x\|=\sup _{k}\left\|\sum_{n=1}^{k} \beta_{n}(x) b_{n}\right\|$. We also make the additional assumption that the basis elements $\left\{b_{n}\right\}$ are adjusted so that $\left\|b_{n}\right\| \leq 1$ (this is always possible). In case $\|\cdot\|$ is actually a norm and $E$ is then a Banach space we can and will assume $\left\|b_{n}\right\|=1$ for $n=1,2, \cdots$.

As usual $E^{*}$ will denote the space of continuous linear functionals on $E$. Then $E^{*}$ is a linear space and the $E$ topology on $E^{*}$, i.e, the weak-star topology, is the topology obtained by taking as a base all sets of the form $N(f, A, \epsilon)=$ $\{g:|f(x)-g(x)|<\epsilon, x \in A\}$ where $f \in E^{*}, A$ is a finite subset of $E$, and $\epsilon>0$.

If $x \in E$ we will define, for $N=1,2, \cdots$,

$$
P_{N} x=\sum_{k=1}^{N} \beta_{k}(x) b_{k}, \quad Q_{N} x=\sum_{k=N+1}^{\infty} \beta_{k}(x) b_{k},
$$

and for $y \in E^{*}, N=1,2, \cdots$, we define

$$
P_{N} y=\sum_{k=1}^{N} \beta_{k}(\cdot) y\left(b_{k}\right) \text {. }
$$


Lemma 2.1. The set $\bigcup_{N=1}^{\infty} P_{N}\left(E^{*}\right)$ is dense in $E^{*}$ with respect to the $E$ topology on $E^{*}$. In fact, if $y \in E^{*}$.there exists a sequence $\left\{y_{N}\right\}$ in $E^{*}$ such that $\left\{y_{N}\right\}$ converges to $y$ in the $E$ topology on $E^{*}$.

Proof. Suppose $y \in E^{*}$. Then for $x \in E$ we have by the continuity of $y$ that $y(x)=\sum_{k=1}^{\infty} \beta_{k}(x) y\left(b_{k}\right)$. Put $y_{N}=\sum_{k=1}^{N} \beta_{k}(\cdot) y\left(b_{k}\right)$. Then $y_{N} \in P_{N}\left(E^{*}\right)$ for $N=1,2, \cdots$ and $\left\{y_{N}\right\}$ converges to $y$ in the $E$ topology on $E^{*}$, so the lemma follows.

The vector space of all sequences of real numbers with the topology of coordinatewise convergence is denoted by $l$. The subset of $l$ consisting of all bounded sequences will be denoted by $l_{\infty}$ and $l_{\infty}^{+}$will symbolize the positive cone of $l_{\infty}$, i.e. $x=\left\{x_{i}\right\} \in l_{\infty}^{+}$iff $x_{i}>0$ for each $i$ and $x \in l_{\infty}$. For $0<p<\infty$ we let $l_{p}$ denote the subset of $l$ consisting of all sequences which have $\|x\|=$ $\left\{\sum_{i=1}^{\infty}\left|x_{i}\right|^{p}\right\}^{1 / p}$ finite where $x=\left\{x_{i}\right\}$. Thus, for $1 \leq p<\infty$, we have $\|\cdot\|$ as the usual norm for $l_{p}$, and for $0<p<1,\|\cdot\|^{p}$ generates an invariant metric under which $l_{p}$ is an $F$-space. The positive cone of $l_{p}$ will be denoted by $l_{p}^{+}$.

3. Bochner's theorem on $E$. Let $\mu$ be a probability measure on the Borel subsets of $E$ where by Borel sets we mean the minimal sigma-algebra containing the open sets. Then the Fourier transform of $\mu$ is a complex-valued function $\phi$ defined on $E^{*}$ (the topological dual of $E$ ) satisfying $\phi(x)=\int_{E} \exp \{i\langle x, y\rangle\} d \mu(y)$ where $\langle x, y\rangle=x(y)$.

If we let $\mathcal{F}$ denote the algebra of cylinder sets, i.e. sets of the form $\{y \in E$ : $\left.\left[\left\langle x_{1}, y\right\rangle, \cdots,\left\langle x_{n}, y\right\rangle\right] \in \Lambda\right\}$ where $x_{1}, \cdots, x_{n} \in E^{*}$ and $\Lambda$ is a Borel subset of $R_{n}$, and $\mathcal{B}(\mathcal{F})$ the minimal sigma-algebra containing $\mathcal{F}$, then it is easy to see that $\phi$ uniquely determines $\mu$ on $\mathfrak{B}(\mathcal{F})$. Now $B(F)$ is contained in the Borel subsets of $E$ and since $E$ is a complete separable metric space it is known that they actually coincide. Hence there is a one-to-one correspondence between probability measures on the Borel subsets of $E$ and their Fourier transforms. To simplify many subsequent statements in the remainder of the paper we assume that all measures are defined on the Borel subsets of $E$.

If $\lambda \in l_{\infty}^{+}$and $\left\{\mu_{\alpha}: \alpha \in A\right\}$ is a family of probability measures on $E$ such that

$$
\mu_{\alpha}\left\{x \in E: \sum_{k=1}^{\infty} \lambda_{k}\left[\beta_{k}(x)\right]^{2}<\infty\right\}=1
$$

for each $\alpha \in A$, we say $\lambda$ is sufficient for the family $\left\{\mu_{a}: \alpha \in A\right\}$. In other words, the map $x \rightarrow\left(\sqrt{\lambda_{1}} \beta_{1}(x), \sqrt{\lambda_{2}} \beta_{2}(x), \cdots\right)$ is an $l_{2}$-valued random variable for each $\mu_{\alpha}$. 
If for each $y \in E$ we have $\sup _{n}\left|\beta_{n}(y)\right|<\infty$, it then follows that any $\lambda \in l_{1}^{+}$ is sufficient for any family of probability measures on $E$.

A family of probability measures $\left\{\mu_{a}: \alpha \in A\right\}$ on $E$ is a $\lambda$-family for some $\lambda \in l_{\infty}^{+}$if $\lambda$ is sufficient for $\left\{\mu_{\alpha}: \alpha \in A\right\}$ and for every $\epsilon, \delta>0$ there is a sequence $\left\{\epsilon_{N}\right\}$ such that

$$
\mu_{\alpha}\left\{x \in E: \sum_{k=N+1}^{\infty} \lambda_{k}\left[\beta_{k}(x)\right]^{2}<\delta\right\}>1-\epsilon
$$

implies

$$
\mu_{a}\left\{x \in E:\left\|\sum_{k=N+1}^{\infty} \beta_{k}(x) b_{k}\right\|<b(\delta)\right\}>1-\left(\epsilon+\epsilon_{N}\right)
$$

where $\lim _{N} \epsilon_{N}=0$ and $b$ is a strictly increasing continuous function on $[0, \infty)$ with $h(0)=0$.

It is quite clear that any family of probability measures on a real separable Hilbert space is a $\lambda$-family with $\lambda=(1,1, \cdots)$ and $b(\delta)=\delta^{1 / 2}$.

Lemma 3.1. If $\left\{\mu_{\alpha}: \alpha \in A\right\}$ is a conditionally compact set of probability measures on $E$ then $\left\{\mu_{a}: \alpha \in A\right\}$ is a $\lambda$-family for every $\lambda \in l_{\infty}^{+}$which is sufficient for $\left\{\mu_{\alpha}: \alpha \in A\right\}$.

Proof. Let $\epsilon, \delta>0$ be given and choose $\lambda \epsilon l_{\infty}^{+}$such that $\lambda$ is sufficient for $\left\{\mu_{\alpha}: \alpha \in A\right\}$. Since $\left\{\mu_{\alpha}: \alpha \in A\right\}$ is conditionally compact there exists a compact set $K$ in $E$ such that $\mu_{\alpha}(K)>1-\epsilon$ for each $\alpha \in A$. Hence there exist points $p_{1}, \cdots, p_{T} \in K$ such that if $S(p, \gamma)=\{x \in E:\|x-p\|<\gamma\}$ then $K \subseteq$ $\bigcup_{i=1}^{T} S\left(p_{i}, \delta / 3\right)$.

Now select $N$ such that $\left\|\sum_{k=N+1}^{\infty} \beta_{k}\left(p_{i}\right) b_{k}\right\|<\delta / 3$ for $i=1, \cdots, T$. Then for $x \in K$ we have $\left\|x-p_{i}\right\|<\delta / 3$ for some $p_{i}$ and hence

$$
\begin{aligned}
\left\|\sum_{k=N+1}^{\infty} \beta_{k}(x) b_{k}\right\| & \leq\left\|\sum_{k=N+1}^{\infty} \beta_{k}(x) b_{k}-\sum_{k=N+1}^{\infty} \beta_{k}\left(p_{i}\right) b_{k}\right\|+\left\|\sum_{k=N+1}^{\infty} \beta_{k}\left(p_{i}\right) b_{k}\right\| \\
& \leq\left\|x-p_{i}\right\|+\left\|\sum_{k=1}^{N}\left[\beta_{k}(x)-\beta_{k}\left(p_{i}\right)\right] b_{k}\right\|+\delta / 3 \leq \delta
\end{aligned}
$$

since $\left\|x-p_{i}\right\|=\sup _{N}\left\|\sum_{k=1}^{N}\left[\beta_{k}(x)-\beta_{k}\left(p_{i}\right)\right] b_{k}\right\|$. Thus there exists an $N$ such that for each $a \in A$

$$
\mu_{\alpha}\left\{x \in E:\left\|\sum_{k=N+1}^{\infty} \beta_{k}(x) b_{k}\right\|<\delta\right\}>1-\epsilon
$$

so clearly $\left\{\mu_{a}: a \in A\right\}$ is a $\lambda$-family. 
Now let $\alpha(\cdot)$ be a convex function on $[0, \infty)$ such that $\alpha(0)=0$ and $\alpha(s)>$ 0 if $s>0$. Further, assume for every compact set $K$ of $E$ there exists an $r>0$ such that $y \in K$ implies $A(y)=\sum_{i=1}^{\infty} \alpha\left[\beta_{i}^{2}(y)\right]<r$, and for every $r>0$ there exists $M>0$ such that $A(y)<r$ implies $\sum_{i=1}^{\infty} \alpha\left[\beta_{i}^{2}(y)\right] \leq M y(\|y\|)$ where $\gamma(\cdot)$ is another continuous function on $[0, \infty)$ such that $\gamma(0)=0$. If the quasi-norm $\|\cdot\|$ on $E$ admits the existence of functions $\alpha(\cdot)$ and $\gamma(\cdot)$ having the above properties we will say that it is accessible. We also note that if $\alpha(\cdot)$ and $\gamma(\cdot)$ satisfy the conditions indicated then $\alpha(\cdot)$ is continuous and strictly increasing on $[0, \infty), \gamma(s)>0$ for $s>0$, and that $\gamma(\cdot)$ can be taken to be increasing on $[0, \infty)$. We note that the accessibility of the quasi-norm on $E$ implies that the map $y \rightarrow\left(\beta_{1}(y), \beta_{2}(y), \ldots\right)$ is continuous from the compact subsets of $E$ into the (topological) subspace $l_{a}$ of $l$ consisting of those sequences $x=\left\{x_{i}\right\}$ for which $\sum_{i=1}^{\infty} a\left(x_{i}^{2}\right)$ is finite. Under additional conditions on $\alpha$ this subspace becomes an Orlicz space (cf. $\$ \zeta$ ).

We now provide some examples of spaces which have an accessible norm. First of all notice that the $l_{p}$ spaces, $2 \leq p<\infty$, have an accessible norm $w$ ith $\alpha(s)=s^{p / 2}$ and $\gamma(s)=s^{p}$. Similarly, if $E=l_{p}, 1 \leq p<2$, then $E$ has an accessible norm with $a(s)=s$ and $\gamma(s)=s^{2}$; if $E=l_{p}, 0<p<1$, then $E$ has an accessible quasi-norm with $\alpha(s)=s$ and $\gamma(s)=s^{2 / p}$. For an example of a space with accessible norm which is not an $l_{p}$ space, consider the Banach space $E$ of real sequences $\left\{x_{i}\right\}$ with norm

$$
\left\|\left\{x_{i}\right\}\right\|^{2}=\sum_{j=1}^{\infty}\left[\sum_{i=2^{j-1}}^{2^{j}-1}\left|x_{i}\right|^{(j+1) / j}\right]^{2 j /(1+j)}
$$

Here $\beta_{i}(x)=x_{i}$, thus if $\alpha(s)=s$ we have

$$
\begin{aligned}
\sum_{j=1}^{\infty} \alpha\left[\beta_{j}^{2}(x)\right] & =\sum_{j=1}^{\infty} x_{j}^{2}=\sum_{j=1}^{\infty} \sum_{i=2^{j-1}}^{2^{j}-1} x_{i}^{2} \\
& \leq \sum_{j=1}^{\infty}\left[\sum_{i=2^{j-1}}^{2^{j}-1}\left|x_{i}\right|^{(j+1) / j}\right]^{2 j /(1+j)}=\left\|\left\{x_{i}\right\}\right\|^{2} .
\end{aligned}
$$

Hence $E$ has an accessible norm if $a(s)=s$ and $\gamma(s)=s^{2}$. Other similar examples follow immediately. For examples of a different type consider those in $\$ 6$.

If the quasi-norm on $E$ is accessible then by the $\tau_{a}$-topology we will mean the topology on $E^{*}$ generated by taking as a subbase all translates of sets of the 
form $\left\{x \in E^{*}: T(x, x)<1\right\}$ as $T(\cdot, \cdot)$ varies over the symmetric, positive definite, bilinear forms on $E^{*}$ which are jointly weak-star sequentially continuous on $E^{*}$ and satisfying $\sum_{k=1}^{\infty} \alpha\left(t_{k k}\right)<\infty$ where $t_{k k}=T\left(\beta_{k}, \beta_{k}\right)$. Bilinear forms having the above properties will be called $\alpha$-operators. In view of Lemma 2.1 and the bilinearity it is easy to see that $T$ is uniquely determined by $t_{i j}=$ $T\left(\beta_{i}, \beta_{j}\right), i, j=1,2, \cdots$.

Lemma 3.2. The sets $\left\{x \in E^{*}: T(x, x)<1\right\}$ and all translates form a basis for the $\tau_{\alpha}$-topology as $T(\cdot, \cdot)$ ranges over the a-operators.

The proof of Lemma 3.2 follows in a standard manner and hence is omitted.

The classical version of Bochner's theorem asserts that a function $\phi(x)$, $x \in R_{n}$, is the Fourier transform of some probability measure on $R_{n}$ iff $\phi$ is positive definite, $\phi(0)=1$, and $\phi$ is continuous at zero. In a real separable Hilbert space $H$ it is possible to introduce a topology $\tau$ (which is determined by certain compact operators) such that a function $\phi$ on $H^{*}=H$ is the Fourier transform of some probability measure on $H$ iff $\phi$ is positive definite, $\phi(0)=1$, and $\phi$ is continuous at zero in the $\tau$-topology. The Hilbert space result is due independently to L. Gross [2] and to V. Sazonov's earlier work [6]. In [3] the work of [2] and [6] is generalized to the $l_{p}$ spaces. Here we will give a Bochner-type theorem in case $E$ has an accessible quasi-norm. Its proof is much like that of Theorem 2.3 of [3] and involves analogues of Lemmas 2.3 and 2.4 of [3]. Due to these similarities the proof of Theorem 3.1 will not be included. However, the formulation of this theorem provided us with some interesting questions and corollaries and it is these that we begin to examine in the following sections.

Theorem 3.1. If $E$ bas an accessible quasi-norm then a function $\phi$ on $E^{*}$ is the Fourier transform of a probability measure iff

(i) $\phi(0)=1, \phi$ is positive definite,

(ii) $\phi$ is continuous in the $\tau_{a}$-topology,

(iii) the family of measures $\left\{\mu_{N}\right\}$ corresponding to $\phi\left(P_{N}(\cdot)\right)$ bas a subsequence which is a $\lambda$-family for some $\lambda \in l_{\infty}^{+}$satisfying $\lim _{k} \sum_{i=k}^{\infty} \lambda_{i} t_{i i}=0$ whenever $\sum_{i=1}^{\infty} \alpha\left(t_{i i}\right)<\infty$. Here $a(\cdot)$, of course, is the function associated with the accessibility of the quasi-norm.

If $E=l_{p}, 2 \leq p<\infty$, then a linear operator $T$ from $l_{p}^{*}$ into $l_{p}$ is an $\mathrm{S}_{p}-o p$ erator if $T$ can be represented as an infinite symmetric positive-definite matrix $\left(t_{i j}\right)$ such that $\sum_{j=1}^{\infty} t_{j j}^{p / 2}<\infty$. Here, by positive definite, we mean that $\sum_{i, j=1}^{n} t_{i j} x_{i} x_{j} \geq 0$ for all $x \in R_{n}$ and all integers $n$. The $\tau_{p}$-topology on $l_{p}^{*}$ is that topology generated by taking as a subbase all translates of all sets of the form $\left\{x \in l_{p}^{*}:\langle T x, x\rangle<1\right\}$ as $T$ varies over the $S_{p}$-operators. In view of Lemma 3.2 , it is easy to see that such sets actually form a basis. Furthermore, our next 
lemma identifies the $\tau_{p}$-topology in terms of a $\tau_{a}$-topology. Its proof is direct and hence is omitted.

Lemma 3.3. If $E=l_{p}, 2 \leq p<\infty$, then the norm of $E$ is accessible with $\alpha(s)=s^{p / 2}, \gamma(s)=s^{p}$ and the $\tau_{a}$-topology on $E^{*}$ is equivalent to the $\tau_{p}$-topology.

Corollary 3.1. If $E=l_{p}, 2 \leq p<\infty$, and $\phi$ is defined on $l_{p}^{*}$ then $\phi$ is the Fourier transform of a probability measure on $l_{p}$ iff

(1) $\phi(0)=1, \phi$ is positive definite,

(2) $\phi$ is $\tau_{p}$-continuous,

(3) the family of measures $\left\{\mu_{N}\right\}$ corresponding to $\left\{\phi\left(P_{N}(\cdot)\right)\right\}$ is a $\lambda$-family for some $\lambda \in l_{p / 2}^{*+}$.

Proof. The sufficiency of (1), (2), (3) follows immediately from Theorem 3.1 and the previous lemma since $\lambda \in l_{p / 2}^{*+}$ implies $\sum_{i=1}^{\infty} \lambda_{i} t_{i i}<\infty$ for all $\left\{t_{i i}\right\}$ satisfying $\sum_{i=1}^{\infty} t_{i i}^{p / 2}<\infty$. Conversely, if $\phi$ is the Fourier transform on $l_{p}^{*}$ of a probability measure on $l_{p}$ then Theorem 3.1 and the previous lemma imply (1), (2) easily and (3) will al so hold for every $\lambda \in l_{p / 2}^{*}$ since $\left\{\mu_{N}\right\}$ is conditionally compact and Lemma 3.1 applies.

4. The continuity theorem. Suppose $l$ is the space of all real sequences with the topology of coordinatewise convergence and $P_{\lambda}(\cdot)$ is the product probability on $l$ such that the $i$ th coordinate is Gaussian with mean zero and variance $\lambda_{i}>$ 0 . If $\mu$ is a measure on $E$ we choose $\lambda \epsilon l_{\infty}^{+}$so that $\lambda$ is sufficient for $\mu$ (such a $\lambda$ always exists if $\sup _{n}\left|\beta_{n}(y)\right|<\infty$ for all $y \in E$ ), and for $x \in l$ we define a "stochastic linear functional" on $E$ in the following manner:

$$
\langle x, y\rangle=\lim _{N} \sum_{k=1}^{N} x_{k} \beta_{k}(y) .
$$

Throughout this section we assume that $\sup _{n}\left|\beta_{n}(y)\right|<\infty$ for all $y \in E$. In case $E$ is a Banach space this is not an additional assumption.

Lemma 4.1. The stochastic linear functional $\langle x, y\rangle=\lim _{N} \sum_{k=1}^{N} x_{k} \beta_{k}(y)$ is Borel measurable on $l \times E$ and if $F=\{(x, y):\langle x, y\rangle$ exists and is finite $\}$ then $\left(P_{\lambda} \times \mu\right)(F)=1$.

The proof of Lemma 4.1 is similar to that of Lemma 3.1 in [3] and, for that reason, is omitted.

If $\mu$ is a measure on the Borel subsets of $E$ we define the extended Fourier transform $\tilde{\phi}(\cdot)$ on $l$ as follows:

$$
\widetilde{\phi}(x)=\int_{E} \exp \{i\langle x, y\rangle\} d \mu(y) \quad(x \in l) .
$$


Then $\tilde{\phi}(\cdot)$ is a Borel measurable function on $l$ which is defined almost everywhere with respect to the measure $P_{\lambda}$. Furthermore, since each $x \in E^{*}$ generates the unique sequence of real numbers $\tilde{x}=\left\{x\left(b_{1}\right), x\left(b_{2}\right), \cdots\right\}$, we may consider $E^{*}$ as a linear subset of $l$, and hence the terminology extended Fourier transform, since for $x \in E^{*}, \phi(x)=\tilde{\phi}(\tilde{x})$.

Theorem 4.1. Let $\left\{\mu_{k}\right\}$ be a sequence of probability measures on $E$ with Fourier transforms $\left\{\phi_{\cdot_{k}}\right\}$. Then $\left\{\mu_{k}\right\}$ converges weakly to a measure $\mu$ with Fourier transform $\phi$ iff $\left\{\mu_{k}\right\}$ is a $\lambda$-family for some $\lambda \in l_{\infty}^{+}$which is also sufficient for $\mu,\left\{\phi_{k}\right\}$ converges to $\phi$ on a subset of $E^{*}$ which is dense in $E^{*}$ with respect to weak-star sequential convergence, and $\left\{\tilde{\phi}_{k}\right\}$ converges in $P_{\lambda}$ measure to $\tilde{\phi}$.

Here again our proof parallels that of Theorem 5.1 of [3] and can be carried out in an analogous manner with appropiate changes in this context. Hence it is omitted. It should be mentioned, however, that the above result generalizes Theorem 5.1 of [3] where $E=l_{p}, 2 \leq p<\infty$. However, it is not quite equivalent to the result of $\mathrm{L}$. Gross (the case $E=l_{2}$ ) but, as the results in $\$ 4$ of [3] indicate, it is the great symmetry of Hilbert space which enables the improved version in [2].

The next corollary is well known and in view of Lemma 3.1 is an immediate corollary to Theorem 4.1 .

Corollary 4.1. Let $\left\{\mu_{k}\right\}$ be a sequence of probability measures on $E$ with Fourier transforms $\left\{\phi_{k}\right\}$. Then $\left\{\mu_{k}\right\}$ converges weakly to a measure $\mu$ iff $\left\{\mu_{k}\right\}$ is conditionally compact and $\left\{\phi_{k}\right\}$ converges on a subset of $E^{*}$ which is dense in $E^{*}$ with respect to weak-star sequential convergence. Further, the Fourier trans. form of $\mu$ is $\phi=\lim \phi_{k}$ on $E^{*}$.

5. Application to Gaussian measures. A probability measure $\mu$ on $E$ is a Gaussian measure with mean vector $a \in E$ if the distribution of the function $\langle y, x\rangle$ is Gaussian with mean $\langle y, a\rangle$ for each $y \in E^{*}$.

For example, if $\left(t_{i j}\right)$ is a positive definite, symmetric matrix satisfying $\sum_{i=1}^{\infty} t_{i i}^{1 / 2}<\infty$ and $E$ is a Banach space with basis $\left\{b_{n}\right\}$, then there exists a unique Gaussian measure $\mu$ with mean zero on $E$ such that

$$
t_{i j}=\int_{E} \beta_{i}(x) \beta_{j}(x) d \mu(x) \quad(i, j=1,2, \cdots) .
$$

Further, the Fourier transform of $\mu$ is $\phi(x)=\exp \{-1 / 2\langle T x, x\rangle\}$ where $T=$ $\left(t_{i j}\right)$ maps $E^{*}$ onto $E$ in such a manner that for $x \in E^{*}$

$$
T x=\sum_{i=1}^{\infty}\left[\sum_{j=1}^{\infty} t_{i j} x\left(b_{j}\right)\right] b_{i}
$$


To see that $T x \in E$ simply observe that

$$
\sum_{i=1}^{\infty} \sum_{j=1}^{\infty}\left|t_{i j}\right| \leq\left[\sum_{i=1}^{\infty} t_{i i}^{1 / 2}\right]^{2}<\infty
$$

and use the fact that $E$ is a Banach space with basis.

Suppose $E$ has an accessible quasi-norm. Then, if $E^{*}$ is such that the sequence $\left\{x_{n}\right\}$ converging to $x$ in the $E$ topology on $E^{*}$ implies $\sup _{n, i}\left|x_{n}\left(b_{i}\right)\right|<$ $\infty$, we will say $E$ has Property $A$.

It is easy to see from the uniform boundedness principle that if $E$ is a Banach space with accessible norm then $E$ has property $A$. Hence the examples of $\$ 3$ all have Property A.

Lemma 5.1. If $E$ has Property $\mathrm{A}$ and $\left\{u_{j}\right\} \in l_{1}^{+}$is such that $\sum_{j=1}^{\infty} a\left(u_{j}\right)<\infty$, then $U(x, y)=\sum_{j=1}^{\infty} u_{j} x\left(b_{j}\right) y\left(b_{j}\right)$ is an $\alpha$-operator on $E^{*}$ and $U(x, x)=0$ iff $x=0$.

The proof of Lemma 5.1 is immediate and hence is omitted.

Lemma 5.2. If $E$ has Property $A$ and $\mu$ is a Gaussian measure on $E$ with mean $a$ then the Fourier transform of $\mu$ is $\exp \{i\langle x, a\rangle-1 / 2 T(x, x)\}$ where $T(\cdot, \cdot)$ is an a-operator on $E^{*}$ such that $T\left(\beta_{i}, \beta_{j}\right)=\int_{E} \beta_{i}(x-a) \beta_{j}(x-a) d \mu(x)$ for $i, j=1,2, \cdots$.

Proof. Let $\phi(x)$ denote the Fourier transform of $\mu$. By Theorem 3.1, $\phi(\cdot)$ is $\tau_{\alpha}$-continuous and also weak-star sequentially continuous (it is a Fourier transform). Hence

$$
\phi(x)=\lim _{N} \phi\left(P_{N} x\right)=\lim _{N} \exp \left\{i\left\langle P_{N} x, a\right\rangle-\frac{1}{2} \sum_{j, k=1}^{N} s_{j k} x\left(b_{j}\right) x\left(b_{k}\right)\right\}
$$

where $P_{N}(x)=\sum_{j=1}^{N} \beta_{j}(\cdot) x\left(b_{j}\right)$ and $s_{j k}=\int_{E} \beta_{j}(y-a) \beta_{k}(y-a) d \mu(y)$. Thus

exists for all $x \in E^{*}$. We define for $x, y \in E^{*}$

$$
S(x, x) \equiv \lim _{N} \sum_{i, j=1}^{N} s_{i j} x\left(b_{i}\right) x\left(b_{j}\right)
$$

$$
S(x, y)=\lim _{N} \sum_{i, j=1}^{N} s_{i j} x\left(b_{i}\right) y\left(b_{j}\right) \text {. }
$$

Since it equals $1 / 2[S(x+y, x+y)-S(x, x)-S(y, y)], S(x, y)$ now exists for all $x, y \in E^{*}$. Further, as is easily seen from the definition, $S(\cdot, \cdot)$ is bilinear on $E^{*}$. We now will show $S(\cdot, \cdot)$ is an $a$-operator and the proof is then completed by setting $T=S$. Since $\phi(x)=\exp \{-1 / 2 S(x, x)\}$ is $\tau_{a}$-continuous there exists an $\alpha$-operator $V$ such that $V(x, x) \leq 1$ implies $S(x, x)<\epsilon<1$. We can (and do) assume $V(x, x)>0$ for all $x \neq 0$ (possible by previous lemma). If $V\left(y_{0}, y_{0}\right)=$ 
$c^{2}>0$ then $V\left(y_{0} / c, y_{0} / c\right)=1$ so $S\left(y_{0} / c, y_{0} / c\right)<\epsilon$ and $S\left(y_{0}, y_{0}\right)<\epsilon c^{2}=$ $\epsilon V\left(y_{0}, y_{0}\right)$. Thus $s_{j j}=S\left(\beta_{j}, \beta_{j}\right)<\epsilon V\left(\beta_{j}, \beta_{j}\right)=\epsilon v_{j j}<v_{j j}$ so $\alpha\left(s_{j j}\right)<\alpha\left(v_{j j}\right)$ since $\alpha(\cdot)$ is increasing. Thus $\sum_{j=1}^{\infty} \alpha\left(s_{j j}\right)<\sum_{j=1}^{\infty} \alpha\left(v_{j j}\right)<\infty$ as $V$ is an $\alpha$-operator. The remaining properties of an $\alpha$-operator are easily seen to hold for $S(\cdot, \cdot)$. For example,

$$
\phi(x)=\exp \{i\langle x, a\rangle-1 / 2 S(x, x)\}
$$

is $\tau_{a}$-continuous on $E^{*}$ and since $\exp \{i\langle x, a\rangle\}$ is $\tau_{a}$-continuous it follows that $S(x, x)$ is $\tau_{a}$-continuous. Hence the lemma holds.

Lemma 5.3. If $E$ bas Property $\mathrm{A}$ and $\left\{\mu_{N}\right\}$ is a sequence of Gaussian measures with mean vectors $\left\{a_{N}\right\}$ such that $\left\{\mu_{N}\right\}$ converges weakly to $\mu$, then $\mu$ is Gaussian with mean $a=\lim _{N} a_{N}$ and

$$
\lim _{N} \sum_{j=1}^{\infty} \alpha\left(\left|t_{j j}^{N}-t_{j j}\right|\right)=0
$$

where $t_{j}^{N}=\int_{E} \beta_{j}^{2}\left(x-a_{N}\right) d \mu_{N}(x), t_{j j}=\int_{E} \beta_{j}^{2}(x-a) d \mu(x)$ for $j=1,2, \cdots$.

Proof. For each measure $\mu_{N}$ we define the measure $\nu_{N}(A)=\mu_{N}(-A)$ and corresponding to $\mu$ we define the measure $\nu(A)=\mu(-A)$. Here, of course, $A$ varies over the Borel subsets of $E$. Then $\left\{\nu_{N}\right\}$ converges weakly to $\nu$. Now $\mu_{N}$ has Fourier transform $\exp \left\{i\left\langle x, a_{N}\right\rangle-1 / 2 T_{N}(x, x)\right\}$ where $T_{N}$ is an a-operator on $E^{*}$ such that $T_{N}\left(\beta_{i}, \beta_{j}\right)=\int_{E} \beta_{i}(x-a) \beta_{j}(x-a) d \mu_{N}(x)$ for $i, j=1,2, \cdots$, and hence $\nu_{N}$ has Fourier transform $\exp \left\{i\left\langle x,-a_{N}\right\rangle-1 / 2 T_{N}(x, x)\right\}$. Thus the sequence of convolution measures $\left\{\mu_{N}^{*} \nu_{N}\right\}$ has Fourier transforms $\exp \left\{-T_{N}(x, x)\right\}$ and, furthermore, they converge weakly to $\mu * \nu$ since $\left\{\mu_{N}\right\}$ and $\left\{\nu_{N}\right\}$ converge weakly to $\mu$ and $\nu$. If $\Gamma_{N}(A)=\left(\mu_{N} * \nu_{N}\right)(\sqrt{2} A)$ for $N=$ $1,2, \cdots$ and $\Gamma(A)=(\mu * \nu)(\sqrt{2} A)$ for Borel sets $A$ in $E$ then $\left\{\Gamma_{N}\right\}$ converges weakly to $\Gamma$ and the Fourier transform of $\Gamma_{N}$ is $\exp \left\{-1 / 2 T_{N}(x, x)\right\}$. If $\delta_{N}$ is the measure which places mass one at $a_{N}$ then $\mu_{N}=\delta_{N} * \Gamma_{N}$ and since both $\left\{\Gamma_{N}\right\}$ and $\left\{\mu_{N}\right\}$ converge weakly to $\Gamma$ and $\mu$ this implies $\left\{\delta_{N}\right\}$ converges weakly to the measure $\delta$ with unit mass at $a=\lim _{N} a_{N}$. To see this last assertion observe that for every $\epsilon>0$ there exists a compact set $K$ in $E$ such that $\mu_{N}(K)>$ $1-\epsilon$ and $\Gamma_{N}(K)>1-\epsilon$ for $N=1,2, \cdots$. Thus

$$
\begin{aligned}
1-\epsilon<\mu_{N}(K) & =\int_{E} \delta_{N}(K-x) d \nu_{N}(x) \\
& \leq \int_{K} \delta_{N}(K-x) d \nu_{N}(x)+\epsilon
\end{aligned}
$$

and hence there exists an $x_{N} \in K$ such that $\delta_{N}\left(K-x_{N}\right)>1-2 \epsilon$. If $\epsilon<1 / 2$ this implies $a_{N} \in K-x_{N}$ and hence we find the sequence $\left\{a_{N}\right\}$ lies in the set of 
differences $C=\{x-y: x, y \in K\}$. Now $C$ is compact, thus $\left\{a_{N}\right\}$ is conditionally compact and since $\left\{\mu_{N}\right\}$ converges to $\mu$ we must have only one limit point for $\left\{a_{N}\right\}$. Thus $\lim _{N} a_{N}=a$ exists and $\left\{\delta_{N}\right\}$ converges weakly to $\delta$, as asserted.

Since $\lim _{N} a_{N}=a$ exists it now follows that $\mu$ is a Gaussian measure (possibly degenerate) with mean $a$ and hence it has Fourier transform $\exp \{i\langle x, a\rangle-1 / 2 T(x, x)\}$ where $T$ is an $\alpha$-operator on $E^{*}$ such that

$$
T\left(\beta_{i}, \beta_{j}\right)=t_{i j}=\int_{E} \beta_{i}(x-a) \beta_{j}(x-a) d \mu(x) \quad(i, j=1,2, \cdots) .
$$

To complete the proof we need only verify (5.2). Now $\left\{\Gamma_{N}\right\}$ converges weakly to $\Gamma$, thus for every $\epsilon>0$ there is a compact set $K$ such that $\Gamma_{N}(K)>1-\epsilon / 2$ for $N=1,2, \cdots$ and hence

$$
1-\exp \left\{-1 / 2 T_{N}(x, x)\right\}=\int_{E}[1-\cos \langle x, y\rangle] d \Gamma_{N}(y)
$$

$$
\leq \frac{1}{2} \int_{K}\langle x, y\rangle^{2} d \Gamma_{N}(y)+\epsilon .
$$

Let $S_{N}(x, x)=1 / 2 \int_{K}\langle x, y\rangle^{2} d \Gamma_{N}(y)$ for $N=1,2, \cdots$. Since $t / 2 \leq 1-e^{-t}, 0 \leq$ $t \leq 1$, (5.3) implies $1 / 4 T_{N}(x, x) \leq S_{N}(x, x)+\epsilon$ provided $T_{N}(x, x) \leq 2$. However, if $T_{N}(x, x)=c^{2}>0$ then $1 / 4 T_{N}(x / c, x / c)=1 / 4 \leq S_{N}(x / c, x / c)+\epsilon \leq 2 S_{N}(x / c, x / c)$ provided $\epsilon<1 / 8$. Thus $T_{N}(x, x) \leq 8 S_{N}(x, x)$ for all $x \in E^{*}$. Hence

$$
t_{j j}^{N}=T_{N}\left(\beta_{j}, \beta_{j}\right) \leq 8 S_{N}\left(\beta_{j}, \beta_{j}\right)=4 \int_{K} \beta_{j}^{2}(y) d \Gamma_{N}(y)=\int_{K} \beta_{j}^{2}(2 y) d \Gamma_{N}(y)
$$

for $N, j=1,2, \cdots$ and we therefore find, since $\alpha$ is convex and the quasi-norm is accessible, that

$$
\begin{aligned}
\sum_{j=1}^{\infty} \alpha\left(t_{j j}^{N}\right) & \leq \sum_{j=1}^{\infty} \int_{K} \alpha\left[\beta_{j}^{2}(2 y)\right] d \Gamma_{N}(y) \\
& =\int_{K} \sum_{j=1}^{\infty} \alpha\left[\beta_{j}^{2}(2 y)\right] d \Gamma_{N}(y) \\
& \leq \int_{K} M \gamma(\|2 y\|) d \Gamma_{N}(y)
\end{aligned}
$$

where $M$ depends only on $K$ and not $N$. That is, since $K$ is compact there exists an $r>0$ such that $\sum_{j=1}^{\infty} \alpha\left[\beta_{j}^{2}(2 y)\right]<r$ for all $y \in K$, and hence there exists an $M>0$ such that $y \in K$ implies

$$
\sum_{j=L+1}^{\infty} a\left[\beta_{j}^{2}(2 y)\right] \leq M \gamma\left(\left\|Q_{L}(2 y)\right\|\right) .
$$

Here $M$ depends only on $K$ through our choice of $r$ and not on $L$. We then have 


$$
\begin{aligned}
\sup _{N} \sum_{j=L+1}^{\infty} a\left(t_{j j}^{N}\right) & \leq \sup _{N} \int_{K} \sum_{j=L+1}^{\infty} a\left[\beta_{j}^{2}(2 y)\right] d \Gamma_{N}(y) \\
& \leq \sup _{N} M \int_{K} \gamma\left(\left\|Q_{L}(2 y)\right\|\right) d \Gamma_{N}(y)
\end{aligned}
$$

and as $L$ approaches infinity $\gamma\left(\left\|Q_{L}(2 y)\right\|\right)$ tends to zero on $K$ boundedly, i.e. $\gamma\left(\left\|Q_{L}(2 y)\right\|\right) \leq \gamma\left(\sup _{y \in K} 2\|2 y\|\right)<\infty$, thus

$$
\lim \sup _{N} \sum_{j=L+1}^{\infty} a\left(t_{j j}^{N}\right)=0 .
$$

We then have the conclusion (5.3) because $\lim _{N} t_{j}^{N}=t_{j j}$ for $j=1,2, \cdots$ and hence for every $\epsilon>0$ and integer $L$ there exists $N$ sufficiently large such that

$$
\begin{aligned}
\sum_{j=1}^{\infty} a\left(\left|t_{j j}^{N}-t_{j j}\right|\right) & \leq \epsilon+\sum_{j=L}^{\infty} a\left(\left|t_{j j}^{N}-t_{j j}\right|\right) \\
& \leq \epsilon+\sum_{j=L}^{\infty} \alpha\left(t_{j j}^{N}\right)+\sum_{j=L}^{\infty} \alpha\left(t_{i j}\right) .
\end{aligned}
$$

Now $\lim _{L} \sum_{j=L}^{\infty} \alpha\left(t_{j j}\right)=0$ since $T$ is an $\alpha$-operator and $\lim _{L} \sup _{N} \sum_{j=L}^{\infty} \alpha\left(t_{j j}^{N}\right)=$ 0 by previous remarks, so (5.4) implies (5.2).

An $F$-space $E$ has an accessible quasi-norm in botb directions if there exist functions $\alpha, \gamma_{1}, \gamma_{2}$ such that $E$ has an accessible quasi-norm with respect to $\alpha$ and $\gamma_{2}$ and, for every $y \in E, \gamma_{1}(\|y\|) \leq \sum_{j=1}^{\infty} \alpha\left(\beta_{j}^{2}(y)\right)$. Here $\gamma_{1}(\cdot)$ is an increasing continuous function on $[0, \infty)$ such that $\gamma_{1}(0)=0, \gamma_{1}(s)>0$ for $s>0$, and $\gamma_{2}, a$ satisfy the conditions required in the definition of an accessible quasinorm. Geometrically, the above inequality implies that the inverse of the map $y \rightarrow\left(\beta_{1}(y), \beta_{2}(y), \cdots\right)$ is continuous from the Orlicz space related to $l_{a}$ into $E$ under additional conditions (cf. (6.3)) on $\alpha$.

Let $E$ be a real separable $F$-space having an accessible quasi-norm in both directions. Then $E$ has Property $B$ provided $E$ has Property $A$ and there is a constant $M>0$ satisfying

$$
\int_{-\infty}^{\infty} \alpha\left(u^{2}\right) d \nu(u) \leq M \alpha\left[\int_{-\infty}^{\infty} u^{2} d \nu(u)\right]
$$

for all Gaussian measures $\nu$ on $(-\infty, \infty)$ with mean zero.

Lemma 5.4. Let $E$ have Property $\mathrm{B}$ and suppose $T$ is an a-operator on $E^{*}$. Then $\phi(x)=\exp \{-1 / 2 T(x, x)\}$ is the Fourier transform of a Gaussian measure $\mu$ on E. Further, $T\left(\beta_{i}, \beta_{j}\right)=\int_{E} \beta_{i}(y) \beta_{j}(y) d \mu(y)$ for $i, j=1,2, \cdots$.

Proof. Let $\mu_{N}$ denote the Gaussian measure on $E$ corresponding to the 
Fourier transform $\exp \left\{-1 / 2 T\left(P_{N} x, P_{N} x\right)\right\}$ for $N=1,2, \cdots$. We show $\left\{\mu_{N}\right\}$ is conditionally compact and hence, by Corollary $4.1,\left\{\mu_{N}\right\}$ converges to the measure $\mu$ with Fourier transform $\phi(x)$. Let $\epsilon, \delta>0$. Then

$$
\begin{aligned}
\mu_{N}\left\{y:\left\|Q_{L}(y)\right\|>\delta\right\} & =\mu_{N}\left\{y: \gamma_{1}\left(\left\|Q_{L}(y)\right\|\right)>\gamma_{1}(\delta)\right\} \\
& \leq \mu_{N}\left\{y: \sum_{j=L+1}^{\infty} a\left(\beta_{j}^{2}(y)\right)>\gamma_{1}(\delta)\right\} \\
& \leq \frac{M}{\gamma_{1}(\delta)} \sum_{j=L+1}^{\infty} \alpha\left[\int_{E} \beta_{j}^{2}(y) d \mu_{N}(y)\right]=\frac{M}{\gamma_{1}(\delta)} \sum_{j=L+1}^{\infty} \alpha\left(t_{j j}^{N}\right) .
\end{aligned}
$$

However, $t_{j j}^{N}=\int_{E} \beta_{j}^{2}(y) d \mu_{N}(y)=t_{j j}$ for $j=1, \cdots, N$ and $t_{j j}^{N}=0$ for $j>N$, thus $\mu_{N}\left\{y:\left\|Q_{L}(y)\right\|>\delta\right\}<\epsilon$ for $N=1,2, \cdots$ provided $L$ is sufficiently large. Proceeding with standard techniques (see, for example, Lemma 2.4 of $[5]$ ) we see $\left\{\mu_{N}\right\}$ is conditionally compact. As remarked previously, Corollary 4.1 now implies $\left\{\mu_{N}\right\}$ converges weakly to a measure $\mu$ with Fourier transform $\phi(x)$, and this completes the proof.

In view of Lemma 3.1 it is easy to see that Theorem 3.1 also easily applies to the previous lemma.

Theorem 5.1. If $E$ bas Property B then $\mu$ is a Gaussian measure with mean a iff the Fourier transform of $\mu$ is

$$
\phi(x)=\exp \{i\langle x, a\rangle-1 / 2 T(x, x)\}
$$

where $T$ is the a-operator on $E^{*}$ satisfying $T\left(\beta_{i}, \beta_{j}\right)=\int_{E} \beta_{i}(y-a) \beta_{j}(y-a) d \mu(y)$ for $i, \jmath=1,2, \cdots$.

Proof. Combine Lemmas 5.2 and 5.4.

Theorem 5.2. If $E$ has Property B and $\left\{\mu_{\lambda}: \lambda \in \Lambda\right\}$ is a family of Gaussian measures with means $a_{\lambda}$ and Fourier transforms

$$
\phi_{\lambda}(x)=\exp \left\{i\left\langle x, a_{\lambda}\right\rangle-1 / 2 T_{\lambda}(x, x)\right\}
$$

then $\left\{\mu_{\lambda}: \lambda \in \Lambda\right\}$ is conditionally compact iff

(1) $\left\{a_{\lambda}: \lambda \in \Lambda\right\}$ is conditionally compact in $E$;

(2) the family $\left\{\phi_{\lambda}\left(P_{N} x\right): \lambda \in \Lambda\right\}$ is equicontinuous on $P_{N} E^{*}$ for $N=1,2$, $\cdots$;

(3) $\lim _{L} \sup _{\lambda} \sum_{j=L+1}^{\infty} \alpha\left(t_{j j}^{\lambda}\right)=0$.

Proof. If $\left\{\mu_{\lambda}: \lambda \in \Lambda\right\}$ is conditionally compact then every sequence in $\left\{\mu_{\lambda}: \lambda \in \Lambda\right\}$ has a weakly convergent subsequence. Consequently, by Lemma 5.3, (1) holds and (2) follows since $\left\{\mu_{\lambda}^{P_{N}}: \lambda \in \Lambda\right\}$ is conditionally compact for $N=$ 
$1,2, \ldots$. Now if $\lim _{L} \sup _{\lambda} \sum_{j=L+1}^{\infty} \alpha\left(t_{j j}^{\lambda}\right)=r>0$ then there exists $\lambda_{1}, \lambda_{2}$, .. such that $\sum_{j=L+1}^{\infty} \alpha\left(t_{j j}^{\lambda k}\right)>r / 2$ for all $L \leq k$. However, the sequence of measures $\left\{\mu_{\lambda_{k}}\right\}$ has a convergent subsequence, so assuming $\left\{\mu_{\lambda_{k}}\right\}$ itself converges weakly to a measure $\mu$ we have by Lemma 5.3 that

$$
\lim _{k} \sum_{j=1}^{\infty} a\left(\left|t_{j j}^{\lambda_{k}}-t_{j j}\right|\right)=0
$$

where $t_{j j}=\int_{E} \beta_{j}^{2}(y-a) d \mu(y)$ for $j=1,2, \ldots$. Thus

$$
\sum_{j=L+1}^{\infty} \alpha\left(t_{j j}^{\lambda_{k}}\right) \leq \sum_{j=L+1}^{\infty} \alpha\left[t_{j j}+\left|t_{j j}^{\lambda_{k}}-t_{j j}\right|\right]
$$

$$
\leq \frac{1}{2} \sum_{j=L+1}^{\infty} a\left(2 t_{j j}\right)+\frac{1}{2} \sum_{j=L+1}^{\infty} a\left(2\left|t_{j j}^{\lambda_{k}}-t_{j j}\right|\right)
$$

where the last inequality follows since $\alpha(\cdot)$ is convex on $[0, \infty)$. Now since $E$ has an accessible quasi-norm in both directions we have, given $y \in E$, that there exists $M>0$ such that $\gamma_{1}(\|y\|) \leq \sum_{j=1}^{\infty} \alpha\left[\beta_{j}^{2}(y)\right] \leq M \gamma_{2}(\|y\|)$ and also that $\gamma_{1}(\|\sqrt{2} y\|) \leq \sum_{j=1}^{\infty} a\left[2 \beta_{j}^{2}(y)\right] \leq M \gamma_{2}(\|\sqrt{2} y\|)$. We now show that $\sum_{j=1}^{\infty} a\left(t_{j j}\right)<\infty$ implies $\sum_{j=1}^{\infty} a\left(2 t_{j j}\right)<\infty$. Let $s_{n}=\sum_{j=1}^{n} t_{j j}^{1 / 2} b_{j}$ for $n=1,2, \ldots$. Then $\left\{s_{n}\right\}$ is a Cauchy sequence in $E$. That is, if $n>k$ then $\left\|s_{n}-s_{k}\right\|=$ $\left\|\sum_{j=k+1}^{n} t_{j j}^{1 / 2} b_{j}\right\|$.

Now $\gamma_{1}\left(\left\|s_{n}-s_{k}\right\|\right) \leq \sum_{j=1}^{\infty} a\left(\beta_{j}^{2}\left(s_{n}-s_{k}\right)\right)$ and since $\beta_{j}^{2}\left(s_{n}-s_{k}\right)=t_{j j}$ for $k+1 \leq j \leq n$ we have $\gamma_{1}\left(\left\|s_{n}-s_{k}\right\|\right) \leq \sum_{j=k+1}^{n} a\left(t_{j j}\right)$, and $\sum_{j=1}^{\infty} \alpha\left(t_{j j}\right)<\infty$ along with the properties of $\gamma_{1}$ implies $\lim _{n, k}\left\|s_{n}-s_{k}\right\|=0$. Thus $\left\{s_{n}\right\}$ converges to a vector $s$ and $\left\{\sqrt{2 s}{ }_{n}\right\}$ converges to $\sqrt{2 s}$ so

$$
\sum_{j=1}^{\infty} a\left(2 t_{j j}\right)=\sum_{j=1}^{\infty} a\left(2 \beta_{j}^{2}(s)\right) \leq M \gamma_{2}(\|\sqrt{2} s\|)<\infty
$$

for some constant $M>0$. We now verify that (5.5) implies

$$
\lim _{k} \sum_{j=1}^{\infty} \alpha\left(2\left|t_{j j}^{\lambda_{k}}-t_{j j}\right|\right)=0 \text {. }
$$

By the above argument the vectors $v_{k}=\sum_{j=1}^{\infty}\left|t_{j j}^{\lambda k}-t_{j j}\right|^{1 / 2} b_{j}$ converge to zero since $\gamma_{1}\left(\left\|v_{k}\right\|\right) \leq \sum_{j=1}^{\infty} a\left(\left|t_{j j}^{\lambda_{k}}-t_{j j}\right|\right)$ and (5.5) implies this last term goes to zero as $k$ goes to infinity. Thus the sequence $\left\{\sqrt{2 v} v_{k}\right\}$ also converges to zero, and hence

$$
0 \leq \lim _{k} \sum_{j=1}^{\infty} a\left(2\left|t_{j j}^{k}-t_{j j}\right|\right) \leq M \lim _{k} \gamma_{2}\left(\left\|\sqrt{2} v_{k}\right\|\right)=0
$$

for some constant $M$, so (5.7) holds. Applying (5.7) and $\sum_{j=1}^{\infty} \alpha\left(2 t_{j j}\right)<\infty$ to (5.6) we see 


$$
\lim \sup _{k} \sum_{j=L+1}^{\infty} \alpha\left(t_{j j}^{\lambda_{k}}\right)=0 .
$$

This contradicts $\sum_{j=L+1}^{\infty} a\left(t_{j j}^{\lambda_{k}}\right)>r / 2$ for all $L \leq k$ and hence (3) holds.

For the converse assume (1), (2), (3) hold. Let $\epsilon, \delta>0$. Then

$$
\begin{aligned}
\mu_{\lambda}\left\{y:\left\|\sum_{j=L+1}^{\infty} \beta_{j}(y) b_{j}\right\|>\delta\right\}=\mu_{\lambda}\left\{y: \gamma_{1}\left(\left\|Q_{L} y\right\|\right)>\gamma_{1}(\delta)\right\} \\
\leq \mu_{\lambda}\left\{y: \sum_{j=L+1}^{\infty} a\left(\beta_{j}^{2}(y)\right)>\gamma_{1}(\delta)\right\} \leq \frac{M}{\gamma_{1}(\delta)} \sum_{j=L+1}^{\infty} a\left(t_{j j}^{\lambda}\right) .
\end{aligned}
$$

Thus by (3) we have for $L$ sufficiently large that $\mu_{\lambda}\left\{y:\left\|Q_{L} y\right\|>\delta\right\}<\epsilon$ for all $\lambda \in \Lambda$. Now arguing as indicated in Lemma 5.4 we see (5.8) and condition (2) implies $\left\{\mu_{\lambda}: \lambda \in \Lambda\right\}$ is conditionally compact, so the theorem is proved.

Theorem 5.3. If $E$ has Property B and $\left\{\mu_{k}\right\}$ is a sequence of Gaussian measures with mean vectors $\left\{a_{k}\right\}$ and Fourier transforms

$$
\phi_{k}(x)=\exp \left\{i\left\langle x, a_{k}\right\rangle-1 / 2 T_{k}(x, x)\right\}
$$

thein $\left\{\mu_{k}\right\}$ converges weakly to a measure $\mu$ iff

(i) $\lim _{k} \phi_{k}(x)$ exists on $\bigcup_{N=1}^{\infty} P_{N}\left(E^{*}\right)$,

(ii) $\lim _{k, n} \sum_{j=1}^{\infty} \alpha\left(\left|t_{j j}^{k}-t_{j j}^{n}\right|\right)=0$.

Further, the measure $\mu$ is Gaussian with mean $a=\lim a_{k}$ and Fourier transform $\phi(x)=\exp \{i\langle x, a\rangle-1 / 2 T(x, x)\}$ where $t_{i j}=T\left(\beta_{i}, \beta_{j}\right)=\int_{E} \beta_{i}(y-a) \beta_{j}(y-a) d \mu(y)$ for $i, j=1,2, \cdots$.

The proof of Theorem 5.3 follows directly by using Lemma 5.3 and the techniques employed in the proof of Theorem 5.2, so it is omitted.

Corollary 5.1. If $E$ bas Property B and $\mu$ is a measure on $E$ such that every linear functional on $E$ bas a Gaussian distribution, then there exists a vector $a$ in $E$ such that $a$ is the mean of $\mu$, i.e. $\int_{E} x(y) d \mu(y)=x(a)$ for every $x \in E^{*}$.

Proof. Since $\mu^{P}$ is a Gaussian measure on $P_{N}(E)$ with mean $a_{N}$ in $P_{N}(E)$ for $N=1,2, \cdots$, and $\left\{\mu^{P} N\right\}$ converges to $\mu$, it follows from Theorem 5.3 that $a=\lim _{N} a_{N}$ exists and the corollary follows.

As a result of Corollary 5.1 we find that in our definition of a Gaussian measure on $E$ we need not assume that a mean vector $a$ exists provided $E$ has Property $B$.

If $E=l_{p}$ and $y=\left(y_{1}, y_{2}, \cdots\right)$ then as is usual $\beta_{j}(y)=y_{j}$ for $j=1,2, \ldots$. 
Corollary 5.2. If $E=l_{p}, 2 \leq p<\infty$, and $\left\{\mu_{k}\right\}$ is a sequence of Gaussian probability measures on $l_{p}$ with mean vectors $\left\{a_{k}\right\}$ and $t_{i j}^{k}=$ $\int_{E} \beta_{i}\left(y-a_{k}\right) \beta_{j}\left(y-a_{k}\right) d \mu_{k}(y)$ for $i, j, k=1,2, \cdots$, then $\left\{\mu_{k}\right\}$ converges weakly to a measure $\mu$ iff

(1) $\lim _{k} a_{k}$ exists;

(2) $\lim _{k} t_{i j}^{k}$ exists for $i, j=1,2, \cdots$;

(3) $\lim _{k, n} \sum_{j=1}^{\infty}\left|t_{j j}^{k}-t_{j j}^{n}\right|^{p / 2}=0$.

Furthermore, $\mu$ is then the Gaussian measure with mean $a=\lim _{k} a_{k}$ and satisfying $t_{i j}=\int_{E} \beta_{i}(y-a) \beta_{j}(y-a) d \mu(y)$ for $i, j,=1,2, \cdots$.

Proof. This follows from Theorem 5.3 since if $E=l_{p}, 2 \leq p<\infty$, then $E$ has Property B. To see this let $\alpha(s)=s^{p / 2}$ and $\gamma_{1}(s)=\gamma_{2}(s)=s^{p}$. Then $l_{p}$ has an accessible norm in both directions with respect to $a, \gamma_{1}, \gamma_{2}$. Further, for every Gaussian measure $\nu$ on $(-\infty, \infty)$ with mean zero we have

$$
\int_{-\infty}^{\infty} \alpha\left(s^{2}\right) d \nu(s)=\int_{-\infty}^{\infty}|s|^{p} d \nu(s)=M \alpha\left[\int_{-\infty}^{\infty} s^{2} d \nu(s)\right]
$$

where $M=\Gamma((p+1) / 2)[\Gamma(3 / 2)]^{-p / 2} \pi^{(p-1) / 2}$ and since $l_{p}$ is a Banach space we see $l_{p}$ has Property $A$. Combining these we find that $l_{p}$ has Property $B$ and that conditions (1), (2), and ( 3 ) are equivalent to conditions (i) and (ii) of Theorem 5.3. Thus the corollary is proved.

It should also be mentioned that (1), (2), (3) are sufficient for $\left\{\mu_{k}\right\}$ to converge weakly if $1 \leq p<2$. The necessity in this case is unknown.

Corollary 5.3. If $E=l_{p}, 2 \leq p<\infty$, then

$$
\phi(x)=\exp \{i(x, a)-1 / 2(T x, x)\} \quad\left(x \in E^{*}\right)
$$

is the Fourier transform of a Gaussian measure on $E$ iff $a \in E$ and $T$ is an $S_{p}$ operator.

Proof. Combine Corollary 5.2 and the ideas used in the proof of Corollary 5.3.

6. As pointed out in the proof of Corollary 5.2 the $l_{p}$ spaces, $2 \leq p<\infty$, each have Property B. It is natural to ask if other spaces have it, or, for that matter, if there are other spaces. which have a quasi-norm which is accessible in both directions. In this section we characterize all possible spaces with quasinorm accessible in both directions provided the function $\alpha(\cdot)$ used in the definition of an accessible quasi-norm satisfies the additional condition

$$
\alpha(2 s) \leq M \alpha(s)
$$

for all $s \geq 0$ and some $M<\infty$. This result is found in Theorem 6.3. Furthermore, if, in addition to (6.1), there is a constant $C$ such that 


$$
\int_{-\infty}^{\infty} \alpha\left(u^{2}\right) d \nu(u) \leq C \alpha\left[\int_{-\infty}^{\infty} u^{2} d \nu(u)\right]
$$

for all Gaussian measures $\nu$ on $(-\infty, \infty)$ with mean zero, then our characterization applies to spaces with Property B. Throughout this section $a(\cdot)$ will always have the usual properties required in the definition of accessibility as well as (6.1). Condition (6.2) will be used only in connection with Property B.

Since $a(\cdot)$ is convex we have for $a, b \geq 0$ that $a(a+b) \leq 1 / 2[a(2 a)+a(2 b)]$ and by $(6.1)$ we find

$$
a(a+b) \leq(M / 2)[a(a)+\alpha(b)]
$$

Now we define $E_{a}$ as the space of real sequences satisfying $\sum_{i=1}^{\infty} \alpha\left(y_{i}^{2}\right)<\infty$. Using (6.3) it follows that $E_{\alpha}$ is a vector space over the reals. If $x, y \in E_{\alpha}$ we define

$$
J(x, y)=\sum_{i=1}^{\infty} \alpha\left(\left(x_{i}-y_{i}\right)^{2}\right) .
$$

Then $J(x, y)$ is invariant under translations and we denote by $\mathcal{G}_{a}$ all subsets $G$ of $E_{\alpha} \times E_{a}$ such that there exists an $r>0$ with the property that $J(x, y)<r$ implies $(x, y) \in G$. Then $\bigodot_{a}$ is a uniformity for $E_{\alpha}[4$, p. 176$]$ and the topology $\tau$ of the uniformity $\oint_{\alpha}$ consists of the family of subsets $A$ of $E_{\alpha}$ such that for each $x \in A$ there is a $G \in \mathcal{S}_{\alpha}$ such that $\{y:(x, y) \in G\} \subseteq A$. Now the subfamily $\mathbb{B}_{a}=$ $\left\{G_{1}, G_{2}, \cdots\right\}$ of $\mathcal{G}_{\alpha}$ where $G_{n}=\{(x, y): J(x, y)<1 / n\}$ for $n=1,2, \ldots$ is a countable symmetric base for $\oint_{a}$ and since $J(x, y)>0$ for $x \neq y$ it follows that the topology $\tau$ is Hausdorff. Hence the metrization theorem [4, p. 185] tell us that $\left(E_{a}, \tau\right)$ is metrizable.

In fact, using the proof and the notation employed in the metrization theorem we will show that there exists an invariant metric $d(\cdot, \cdot)$ on $E_{a^{\prime}}$ i.e. $d(x, y)=$ $d(x+z, y+z)$ for all $z \in E_{a}$, such that $d($, ) generates the topology $\tau$, the quasi-norm $\|x\|=d(0, x)$ is accessible in both directions, and that $E_{a}$ is an $F$ space with respect to $\tau$.

Linking our situation to the metrization theorem we let $U_{0}=E_{a} \times E_{a}, U_{1}=$ $\{(x, y): J(x, y)<1\}$, and $U_{n}=\left\{(x, y): J(x, y)<\left(9 M^{4}\right)^{-n+1}\right\}$ for $n=1,2, \cdots$ where $M$ is as in (6.1). Then defining $d($,$) as in [4, p. 185] we have that d($, is a metric satisfying

$$
U_{n} \subseteq\left\{(x, y): d(x, y)<2^{-n}\right\} \subseteq U_{n-1}
$$

for $n=1,2, \cdots$ and that $d(x, y) \leq 1 / 2$ for all $x, y \in E_{a}$. Moreover, examining the definition of $d($,$) we find d($,$) is invariant since our sets \left\{U_{n}\right\}$ are invariant in 
the sense that $(x, y) \in U_{n}$ iff $(x+z, y+z) \in U_{n}$ for every $z \in E_{\alpha}$. In view of (6.5) if $x \in E_{\alpha}$ then $(x, 0) \in U_{n-1}-U_{n}(n=2,3, \cdots)$ iff $\Gamma^{n-1} \leq J(x, 0)<\Gamma^{n-2}$ and $2^{-n-1} \leq d(x, 0)<2^{-n+1}$ where $\Gamma=\left(9 M^{4}\right)^{-1}$. Hence if $\gamma_{1}(s), \gamma_{2}(s)$ are continuous strictly increasing functions on $[0, \infty)$ such that $\gamma_{i}(0)=0$ for $i=1,2$, and satisfying

$$
\gamma_{2}\left(2^{-n-1}\right) \geq \Gamma^{n-2}, \quad \gamma_{1}\left(2^{-n+1}\right) \leq \Gamma^{n-1}
$$

for $n=1,2,3, \cdots$ we have

$$
\gamma_{1}(d(x, 0)) \leq J(x, 0) \leq \gamma_{2}(d(x, 0))
$$

provided $(x, 0) \in U_{1}$, i.e. $J(x, 0)<1$. If $(x, 0) \in U_{0}-U_{1}$ then $J(x, 0) \geq 1,1 / 2 \geq$ $d(x, 0) \geq 1 / 4$, and since $\gamma_{2}(1 / 4) \geq \Gamma^{-1}=9 M^{4}, \gamma_{1}(1) \leq 1$ we have

$$
\gamma_{1}(d(x, 0)) \leq J(x, 0) \leq M_{1} \gamma_{2}(d(x, 0))
$$

provided $J(x, 0) \leq M_{1} \Gamma^{-1}$.

Let $c>0$ be such that $\gamma_{1}^{-1}\left(\alpha\left(c^{2}\right)\right) \leq 1$.

Theorem 6.1. The metric space $\left(E_{\alpha}, \tau\right)$ with quasi-norm $\|x\|=d(x, 0)$ as above is an F-space with basis $\left\{b_{n}\right\}$. Here $b_{n}$ is the vector with $c$ as the nth coordinate and all other coordinates zero. Further, $\left\|b_{n}\right\| \leq 1$ for $n=1,2, \cdots$ and the quasi-norm is accessible in both directions with respect to the functions $\alpha, \gamma_{1}, \gamma_{2}$ where $\gamma_{1}, \gamma_{2}$ are as in (6.6).

To show $\left(E_{\alpha}, \tau\right)$ is an $F$-space we first must show that it is complete in the metric $d($,$) and that it is also a linear topological space in the topology \tau$. Then the assertions about $\left\{b_{n}\right\}$ are immediate in view of (6.8), the definition of $E_{\alpha}$, and our choice of $c>0$. Finally, the accessibility of the quasi-norm will follow from (6.7) and (6.8) provided we can show that for any compact set $K$ of $\left(E_{a}, r\right)$ there is an $r<\infty$ such that $K \subseteq\{x: J(x, 0)<r\}$. These facts will be obtained in the next several lemmas and hence Theorem 6.1 holds.

Lemma 6.1. The metric space $\left(E_{a}, \tau\right)$ with the invariant metric $d(\cdot, \cdot)$ as defined above is a complete metric space.

The proof of Lemma 6.1 follows in exactly the same manner as in the $l_{p}$ spaces and hence is omitted.

Lemma 6.2. $\left(E_{a}, \tau\right)$ is a linear topological space.

Proof. Since $\left(E_{\alpha}, \tau\right)$ is a complete metric space in the invariant metric $d($,$) it suffices to show by Theorem 12$ of $[1$, p. 53] that the mapping $\psi(b, x)=$ $b x$ from $R \times E_{a}$ into $E_{a}$ is continuous in $b$ for each $x$ and continuous in $x$ for each $b$. First fix $x$ and choose $\left\{b_{n}\right\}$ in $R$ converging to $b$. Then 


$$
\gamma_{1}\left(d\left(\left(b-b_{n}\right) x, 0\right)\right) \leq J\left(\left(b-b_{n}\right) x, 0\right)=\sum_{j=1}^{\infty} a\left[\left(b-b_{n}\right)^{2} x_{j}^{2}\right]<\infty,
$$

and as $n$ approaches infinity the last term converges to zero by the dominated convergence theroerm since $\left(b-b_{n}\right)^{2} x_{i}^{2} \leq x_{i}^{2}$ for $\left(b-b_{n}\right)^{2}<1$ and $a(\cdot)$ is increasing. Now fix $b$ and choose $\left\{p_{n}\right\}$ converging to $x$ in $E_{a}$. Then, if $k$ is an integer greater than $b^{2}$ we find

$$
\gamma_{1}\left(d\left(b p_{n}, b x\right)\right) \leq J\left(b p_{n}, b x\right)=\sum_{j=1}^{\infty} a\left(b^{2}\left(x_{n, j}-x_{j}\right)^{2}\right)
$$

$$
\leq \sum_{j=1}^{\infty} \alpha\left(k\left(x_{n, j}-x_{j}\right)^{2}\right) \leq M^{k} \sum_{j=1}^{\infty} \alpha\left(\left(x_{n, j}-x_{j}\right)^{2}\right) \leq M^{k} \gamma_{2}\left(d\left(p_{n}-x, 0\right)\right)
$$

since $\left\{p_{n}\right\}$ converging to $x$ implies that $d\left(p_{n}-x, 0\right)<1 / 4$ for $n$ sufficiently large. Now $\lim _{n} \gamma_{2}\left(d\left(p_{n}-x, 0\right)\right)=0$ so $\lim _{n} d\left(b p_{n}, b x\right)=0$ and hence the lemma is proved.

Lemma 6.3. If $J(y, x)$ is defined as in (6.4) and $K$ is a compact subset of $\left(E_{\alpha}, \tau\right)$ then there exists an $r>0$ such that $K \subseteq\{y: J(y, 0)<r\}$.

Proof. If no such $r$ exists there is a seque nce $\left\{y_{n}\right\}$ in $K$ such that $\left\{y_{n}\right\}$ converges to $y$ and $J\left(y_{n}, 0\right)>n$. This contradicts the fact that $\sup _{n} J\left(y_{n}, 0\right)$ is bounded for Cauchy sequences and hence such an $r$ exists so the lemma is proved.

Lemma 6.4. If $\left\{b_{n}\right\}$ is defined as in Theorem 6.1 then $\left\{b_{n}\right\}$ is a basis for $\left(E_{a}, \tau\right)$ and $\left\|b_{n}\right\| \leq 1$ for $n=1,2, \cdots$.

Proof. Combine the definition of $E_{a}$ and $c>0$ along with (6.8).

At this point we have now proved Theorem 6.1. The impact of Theorem 6.1 is that any function $\alpha(\cdot)$ satisfying (6.1) and the usual conditions we have imposed can be used to produce an $F$-space which has an accessible quasi-norm in both directions. We now proceed to characterize these spaces as well as those with Property B.

If $\alpha(t)$ is a convex function on $[0, \infty)$ such that $\alpha(0)=0, \alpha(t)>0$ for $t>0$, then, as mentioned previously, $\alpha(t)$ is also continuous and strictly increasing on $[0, \infty)$. Further, $\Gamma(t)=\alpha\left(t^{2}\right)$ then has the same properties and it follows that $\Gamma(t)=\int_{0}^{t} \rho(s) d s$ where $\rho(0)=0$ and $\rho(s)$ is nondecreasing on $[0, \infty)$. We assume, without loss of generality, that $\rho(s)$ is left continuous and define $\Lambda(t)=\int_{0}^{t} \psi(s) d s$ where $\psi(s)$ is the inverse of $\rho(s)$ on $[0, \infty)$ defined with the understanding that $\psi(s)$ is left continuous where it is finite and that if $\rho(t)$ makes a jump at $t=a$ then $\psi(s)=a$ for $\rho(a-)<s \leq \rho(a+)$, while, if $\rho(t)=c$ for $a<u \leq b$ but $\rho(t)<$ $c$ for $t<a$ then $\psi(c)=a$. Furthermore, $\psi(0)=0$ and if $\lim _{t \rightarrow \infty} \rho(t)=\gamma<\infty$ then 
$\psi(s)=+\infty$ for $s>\gamma$. Then $\Gamma$ and $\Lambda$ are complementary in the sence of Young $[10$, p. 77$]$ and by $\mathcal{S}_{\Gamma}^{*}$ we mean all real sequences $\left\{x_{i}\right\}$ such that

$$
\sum_{i=1}^{\infty} \Gamma\left(\left|x_{i}\right|\right)=\sum_{i=1}^{\infty} \alpha\left(x_{i}^{2}\right)<\infty .
$$

Similarly, $\mathfrak{S}_{\mathbf{\Lambda}}^{*}$ is all sequences such that $\sum_{i=1}^{\infty} \Lambda\left(\left|x_{i}\right|\right)<\infty$.

If $x=\left\{x_{i}\right\}$ is a sequence we define

$$
\begin{aligned}
& \|x\|_{\Gamma}=\sup _{y}\left\{\sum_{i=1}^{\infty}\left|x_{i} y_{i}\right|: \sum_{i=1}^{\infty} \Lambda\left(\left|y_{i}\right|\right) \leq 1\right\}, \\
& \|x\|_{\Lambda}=\sup _{y}\left\{\sum_{i=1}^{\infty}\left|x_{i} y_{i}\right|: \sum_{i=1}^{\infty} \Gamma\left(\left|y_{i}\right|\right) \leq 1\right\} .
\end{aligned}
$$

The Orlicz space $\mathfrak{S}_{\boldsymbol{\Gamma}}\left(\mathfrak{S}_{\boldsymbol{\Lambda}}\right)$ is the collection of all real sequences such that $\|x\|_{\boldsymbol{\Gamma}}$ $\left(\|x\|_{\Lambda}\right)$ is finite. Since $\alpha(t)$ satisfies $(6.1)$ it follows that $\Gamma(2 t)=\alpha\left(4 t^{2}\right) \leq$ $M \alpha\left(2 t^{2}\right) \leq M^{2} \alpha\left(t^{2}\right)=M^{2} \Gamma(t)$ and hence we know [10, p. 81$]$ that $\mathcal{S}_{\Gamma}=\mathcal{S}_{\Gamma}^{*}$. Further, it is known that $\mathcal{S}_{\Gamma}=\mathcal{S}_{\Gamma}^{*}$ is a real separable Banach space in the norm $\|x\|_{\Gamma}$ and since $\Gamma(2 t) \leq M^{2} \Gamma(t)$ for $t \geq 0$ we also have that $\left\{p_{n}\right\}$ converges to $p$ in norm provided

$$
\lim _{n} \sum_{i=1}^{\infty} \Gamma\left(\left|x_{i, n}-x_{i}\right|\right)=\lim _{n} \sum_{i=1}^{\infty} \alpha\left(\left(x_{i, n}-x_{i}\right)^{2}\right)=0 .
$$

These results follow as in [10, pp. 82-85]. Now it is clear that $E_{a}=\mathcal{S}_{\Gamma}^{*}$ and since $\mathcal{S}_{\Gamma}^{*}=\mathcal{S}_{\Gamma}$ we have $E_{a}=\mathcal{S}_{\boldsymbol{\Gamma}}$. Consequently, $E_{\alpha}=\mathcal{S}_{\boldsymbol{\Gamma}}$ is an $F$-space in the topology $\tau$ and in the topology $\tau_{\Gamma}$ generated by the norm $\|x\|_{\Gamma}$. Further, $\tau_{\Gamma} \subseteq \tau$ since $\left\{p_{n}\right\}$ converging to $p$ with respect to the $\tau$ topology implies $\lim _{n} d\left(p_{n}, p\right)=0$, and hence (6.10) holds so $\left\{p_{n}\right\}$ converges to $p$ in the norm $\|\cdot\|_{\Gamma}$, i.e. in the $\tau_{\Gamma}$ topology. Thus any closed set with respect to $\tau_{\Gamma}$ is also closed with respect to $\tau$ and hence $\tau_{\boldsymbol{r}} \subseteq \tau$ as indicated.

Theorem 6.2. The metric space $\left(E_{\alpha}, \tau\right)$ is a Banach space in the norm $\|\cdot\|_{\Gamma}$.

Proof. Since the topologies $\tau$ and $\tau_{p}$ both make $E_{a}=\delta_{\Gamma}$ an $F$-space and they are comparable it follows that they are identical $[1$, p. 58]. Thus the theorem follows since $\left(E_{\alpha}, \tau_{\Gamma}\right)$ is a Banach space.

Corollary 6.1. The metric space $\left(E_{a}, \tau\right)$ with quasi-norm $\|\cdot\|$ and basis $\left\{b_{n}\right\}$ as defined in Theorem 6.1 is sucb that

(1) If $K$ is any compact subset of $E_{a}$ and $\left\{\beta_{n}\right\}$ represents the coefficient functionals relative to the basis $\left\{b_{n}\right\}$, then $\sup _{n, y \in K}\left|\beta_{n}(y)\right|<\infty$.

(2) The sigma-algebra generated by the weakly open subsets of $E_{\alpha}$ is equal to the sigma-algebra generated by the r-open subsets of $E_{a}$. 
(3) $\left(E_{a}, \tau\right)$ has Property A.

(4) If $\alpha(\cdot)$ also satisfies (6.2), then $\left(E_{\alpha}, \tau\right)$ bas Property B.

Proof. If $K$ is compact then there exists an $r>0$ such that $y \in K$ implies $J(y, 0)<r$. Now, $\beta_{n}(y)=y_{n} / c$ where $y_{n}$ is the $n$th coordinate of $y$ and $c>0$ is the constant chosen as in Theorem 6.1. Further, $\alpha\left(y_{n}^{2} / c^{2}\right)<r$ and hence $\left|y_{n} / c\right| \leq$ $\left\{\alpha^{-1}(r)\right\}^{1 / 2}$ so $\sup _{y \in K, n}\left|\beta_{n}(y)\right| \leq\left\{\alpha^{-1}(r)\right\}^{1 / 2}<\infty$ and (1) holds.

To see that (2) holds combine Theorem 6.2 and the remarks made in the second paragraph of $\$ 3$.

To prove $\left(E_{a}, \tau\right)$ has Property $A$ we note that Theorem 6.1 implies $d($,$) de-$ fines an accessible quasi-norm for $\left(E_{a}, \tau\right)$, and by Theorem 6.2 that $\left(E_{\alpha}, \tau\right)$ is also a Banach space in the norm $\|\cdot\|_{\Gamma}$. Hence $\left\{x_{n}\right\}$ in $E_{\alpha}^{*}$ converging to $x$ in the $E_{a}$ topology on $E_{a}^{*}$ implies $\sup _{n, i}\left|x_{n}\left(b_{i}\right)\right|<\infty$ provided $\sup _{i}\left\|b_{i}\right\|_{\Gamma}<\infty$. Now $\left\|b_{i}\right\|_{\Gamma}=\sup _{y}\{|c y|: \Lambda(|y|) \leq 1\}$ for $i=1,2, \cdots$ and this last quantity is finite, so $\sup _{i}\left\|b_{i}\right\|_{\Gamma}<\infty$ as desired.

Condition (4) now follows from (3) and the definition of Property B.

Theorem 6.3. If $E$ bas a quasi-norm which is accessible in both directions with respect to the functions $\alpha, \rho_{1}, \rho_{2}$ and $\alpha(\cdot)$ satisfies condition (6.1), then $E$ and the Orlicz space $E_{a}$ are bomeomorphic and isomorphic.

Proof. We denote the quasinorm on $E$ by $\|\cdot\|$. If $p \in E$ we let $\Phi(p)=$ $\left(\beta_{1}(p), \beta_{2}(p), \cdots\right)$. Since $\|\cdot\|$ is accessible, $p \in E$ implies $\sum_{j=1}^{\infty} \alpha\left[\beta_{j}^{2}(p)\right]<$ $\infty$, thus $\Phi(p) \in E_{\alpha}$. On the other hand, if $\left(c_{1}, c_{2}, \cdots\right) \in E_{a}$, then $\sum_{i=1}^{\infty} a\left(c_{i}^{2}\right)$ $<\infty$ and if $p_{n}=\sum_{i=1}^{n} c_{i} b_{i}$ for $n=1,2, \ldots$ then $\left\{p_{n}\right\}$ is a Cauchy sequence in $E$. Let $p=\lim _{n} p_{n}$. Then $\Phi(p)=\left(\beta_{1}(p), \beta_{2}(p), \cdots\right)=\left(c_{1}, c_{2}, \cdots\right)$ so $\Phi$ is a one-to-one map of $E$ onto $E_{a}$ which clearly preserves the algebraic relations. To see that $\Phi$ is also a homeomorphism we first note that since $\|\cdot\|$ is accessible in both directions it follows that a sequence $\left\{p_{n}\right\}$ in $E$ converges to zero iff $\lim _{n} \sum_{j=1}^{\infty} \alpha\left[\beta_{j}^{2}\left(p_{n}\right)\right]=0$. Then, if $\Phi\left(p_{n}\right)=\left(\beta_{1}\left(p_{n}\right)\right.$, $\left.\beta_{2}\left(p_{n}\right), \cdots\right)$ we see from previous considerations that $\left\{\Phi\left(p_{n}\right)\right\}$ converges to zero in the norm on $E_{a}$ iff $\lim _{n} \sum_{j=1}^{\infty} \alpha\left[\beta_{j}^{2}\left(p_{n}\right)\right]=0$. Consequently, $\Phi$ is a homeomorphism and the theorem is proved.

Corollary 6.2. If $E$ bas a quasi-norm which is accessible in both directions with respect to the functions $\alpha, \rho_{1}, \rho_{2}$ and $\alpha(\cdot)$ satisfies condition (6.1), then $E$ is isomorphic and bomeomorphic to $l_{p}$ for some $p, 2 \leq p<\infty$, iff

$$
\varlimsup_{t \downarrow 0} \frac{\alpha\left(t^{2}\right)}{t^{p}}=k<\infty
$$




$$
\frac{\lim }{t ! 1} \frac{\alpha\left(t^{2}\right)}{t^{p}}=l>0
$$

Proof. If (6.11) and (6.12) hold then $E$ is isomorphic to $l_{p}$ with respect to the map $\Phi(x)=\left(\beta_{1}(x), \beta_{2}(x), \cdots\right)$. In view of $(6.11)$ a sequence $\left\{x_{n}\right\}$ converges to zero in $E$ if

$$
\lim _{n} \sum_{j=1}^{\infty}(k+\epsilon)\left|\beta_{j}\left(x_{n}\right)\right|^{p}=0
$$

Thus $\left\{x_{n}\right\}$ converges to zero in $E$ if $\left\{x_{n}\right\}$ converges to zero in $l_{p}$ for some $p$ such that (6.11) holds. On the other hand, if $\left\{x_{n}\right\}$ converges to zero in $E$ then by (6.12) it follows that $\left\{x_{n}\right\}$ converges to zero in $l_{p}$. Thus if (6.11) and (6.12) hold we also find $E$ and $l_{p}$ are homeomorphic as well as isomorphic.

Conversely, if $E$ is homeomorphic and isomorphic to $l_{p}$ then

$$
\lim _{n} \sum_{k=1}^{\infty} a\left(\beta_{k}^{2}\left(x_{n}\right)\right)=0 \quad \text { iff } \quad \lim _{n} \sum_{k=1}^{\infty}\left|\beta_{k}\left(x_{n}\right)\right|^{p}=0
$$

for a sequence of points $\left\{x_{n}\right\}$ in $E=l_{p}$. If $\overline{\lim }_{t 10} \alpha\left(t^{2}\right) / t^{p}=\infty$ we choose $0<$ $\epsilon_{n}<1$ such that $\alpha\left(\left[\epsilon_{n} / n^{1 / p}\right]^{2}\right)>\epsilon_{n}^{p}$ and let $k_{n}$ be such that $1<k_{n}\left(\epsilon_{n}\right)^{p}<2$. Then for $n=1,2, \cdots$ we define the point $x_{n}$ in $E$ such that $\beta_{k}\left(x_{n}\right)=0$ if $k>$ $k_{n}$ and $\beta_{k}\left(x_{n}\right)=\epsilon_{n} / n^{1 / p}$ for $1 \leq k \leq k_{n}$. Thus

$$
\sum_{k=1}^{\infty} a\left(\beta_{k}^{2}\left(x_{n}\right)\right) \geq \sum_{k=1}^{k}\left|\epsilon_{n}\right|^{p}>1, \quad \sum_{k=1}^{\infty}\left|\beta_{k}\left(x_{n}\right)\right|^{p}=\frac{k_{n}\left|\epsilon_{n}\right|^{p}}{n}<\frac{2}{n},
$$

so (6.13) is violated. If $\underline{\lim }_{t \downarrow 0} a\left(t^{2}\right) / t^{p}=0$ there exists $0<\epsilon_{n}<1$ such that $\alpha\left(\epsilon_{n}^{2}\right)<\epsilon_{n}^{p} / n$ for $n=1,2, \ldots$. Let $k_{n}$ be an integer such that $1<k_{n}\left(\epsilon_{n}\right)^{p}<2$ and define the point $x_{n}$ in $E$ such that $\beta_{k}\left(x_{n}\right)=0$ if $k>k_{n}$ and $\beta_{k}\left(x_{n}\right)=\epsilon_{n}$ for $1 \leq k \leq k_{n}$. Then $1<\sum_{k=1}^{\infty}\left|\beta_{k}\left(x_{n}\right)\right|^{p}=k_{n} \epsilon_{n}^{p}<2$ but $\sum_{k=1}^{\infty} a\left(\beta_{k}^{2}\left(x_{n}\right)\right) \leq$ $k_{n} \epsilon_{n}^{p} / n<2 / n$, so (6.13) is again violated. Combining the above we see that the theorem is proved.

Now Theorem 6.3 points out that $E$ is an Orlicz space provided $E$ has a quasi-norm which is accessible in both directions with respect to the functions $\alpha, \rho_{1}, \rho_{2}$ and $\alpha(\cdot)$ satisfies condition (6.1). Furthermore, Corollary 6.2 gives necessary and sufficient conditions for $E$ to be $l_{p}$ for some $p, 2 \leq p<\infty$. For an example of a space $\left(E_{a}, \tau\right)$ constructed as in Theorem 6.1 which is not an $l_{p}$ space, consider $E_{a}$ where $a(t)=0$ for $t=0, a(t)=t^{2}[-\log t]$ for $0<t \leq 1 / e^{2}$, and $a(t)=2 t^{2}$ for $t>1 / e^{2}$. In fact, by the previous corollary, $E_{\alpha} \neq l_{p}$ for any $p, 2 \leq p<\infty$, since $\lim _{t \downarrow 0} \alpha\left(t^{2}\right) / t^{4}=+\infty$ and $\lim _{t \downarrow 0} \alpha\left(t^{2}\right) / t^{4-\delta}=0$ for any 
$\delta>0$. Now further calculation also shows that $E_{a}$, in this case, also has Property $B$. Hence we have an example of a space which satisfies our most rigid requirements and is something other than an $l_{p}$ space, $2 \leq p<\infty$.

Corollary 6.3. If $E$ has Property B and the function $\alpha(\cdot)$ satisfies (6.1), then $\alpha(\cdot)$ also satisfies (6.2) and $E$ is bomeomorphic and isomorphic to the Orlicz space $E_{a}$.

The proof of Corollary 6.3 follows immediately by combining the results of Corollary 6.1 and Theorem 6.3.

7. Infinitely divisible measures on spaces with Property B. The study of infinitely divisible measures on a real separable Hilbert space is due to S.R.S. Varadhan [11], and here we consider similar questions for $F$-spaces with Property $B$ where the function $\alpha(\cdot)$ satisfies (6.1) and (6.2). In view of Theorem 6.3 and Corollary 6.3 it follows that we are actually studying infinitely divisible measures on the Orlicz space $E_{a}$. Furthermore, as pointed out in the proof of Corollary 5.2 , the $l_{p}$ spaces, $2 \leq p<\infty$, each have Property B, so our results will extend those of Varadhan. It should be mentioned, however, that Varadhan's work is used at several points throughout this section.

Throughout the section $E_{a}$ will denote the Hilbert space $l_{2}$ or an $E_{a}$ space where $\alpha(\cdot)$ satisf ies (6.1), (6.2), and if $\alpha_{c}(\cdot)$ is the complementary function of $\alpha(\cdot)$ in the sense of Young [10, p. 77], then $\alpha_{c}(\cdot)$ should also satisfy (6.1). Notice that if $E_{\alpha}=l_{2}$ then a natural choice for the function $\alpha$ is $\alpha(t)=t$. Hence $a_{c}(s)=0$ on $[0,1]$ but $\alpha_{c}(s)=\infty$ for $s>1$. Thus $a_{c}(\cdot)$ does not satisfy (6.1) when $E_{a}=l_{2}$ and this is a special case outside our general setup. Nevertheless, it is easily handled.

In terms of the results of $\$ 6$ we have that $E_{a}$ is equivalent to the Orlicz space $S_{\Gamma}$ where $\Gamma(t)=a\left(t^{2}\right)$. We will let $S_{\alpha}, S_{a_{c}}$ denote the Orlicz spaces given by $a(\cdot)$ and $a_{c}(\cdot)$, respectively. Then the dual space of $S_{a}$ can be identified as $S_{a_{c}}$ and since $\alpha_{c}(\cdot)$ also satisfies (6.1), except when $E_{a}=l_{2}$, it follows that the dual of $S_{a_{c}}$ is $S_{a}$ [10, p. 150].

To simplify our'notation we will use the sequence space representation for all of the spaces of the remainder of the paper.

The following terminology is used. If $F(\cdot)$ is a finite Borel measure on $E_{a}$ then $e(F)$ is the probability measure defined by $\exp \left\{-F\left(E_{a}\right)\right\} \cdot \sum_{k=0}^{\infty} F^{k} / k$ ! where $F^{k}$ is $F$ convolved $k$ times and $F^{0}=\delta_{0}$ where $\delta_{x}$ is the unit mass at $x$. A family $\mathcal{F}$ of probability measures on $E_{a}$ is shift-compact if for every sequence $\left\{\nu_{n}\right\}$ in $\mathcal{F}$ there exists a sequence $\left\{x_{n}\right\}$ in $E_{a}$ such that $\left\{\nu_{n} * \delta_{x_{n}}\right\}$ is compact.

For each vector $\lambda=\left(\lambda_{1}, \lambda_{2}, \cdots\right)$ in the positive cone of $S_{a_{c}}$ which is of norm at most one-half, we define the Hilbert space $H_{\lambda}$ as all sequences $x=$ 
$\left(x_{1}, x_{2}, \cdots\right)$ such that $\|x\|_{\lambda}^{2}=\sum_{i=1}^{\infty} \lambda_{i} x_{i}^{2}<\infty$. In the special case $E_{a}=l_{2}$ we have $S_{a_{c}}=l_{\infty}$ and for simplicity we take $\lambda=(1,1, \cdots)$. Then $H_{\lambda}=l_{2}$ and we shall assume without loss of generality that $\alpha(t) \equiv t$.

Lemma 7.1. $E_{a}$ is a Borel subset of $H_{\lambda}$ for each $\lambda$ in the positive cone of $S_{a_{c}}$. Furthermore, every Borel subset of $E_{a}$ is a Borel subset of $H_{\lambda}$.

Proof. If $E_{a}=l_{2}$ and $\lambda=(1,1, \cdots)$ the result is obvious. In general to see $E_{\alpha} \subseteq H_{\lambda}$ we need only notice that by Young's inequality [10, p. 77] $\lambda_{i} x_{i}^{2} \leq$ $\alpha_{c}\left(\lambda_{i}\right)+\alpha\left(x_{i}^{2}\right)$. Hence for $x=\left(x_{1}, x_{2}, \cdots\right)$ in $E_{a}$ and $\lambda$ as indicated, we have $x \in H_{\lambda}$. To show that the Borel subsets of $E_{a}$ also are Borel subsets of $H_{\lambda}$, we need only show that the norm $\|\cdot\|_{\Gamma}$ is a Borel function on $H_{\lambda}$. Now

$$
\|x\|_{\Gamma}=\sup _{y}\left\{\sum_{i=1}^{\infty}\left|x_{i} y_{i}\right|: \sum_{i=1}^{\infty} \Lambda\left(\left|y_{i}\right|\right) \leq 1\right\}
$$

where $\Lambda$ is the function complementary to $\Gamma$ in the sense of Young. Since $\Gamma(t)=$ $\alpha\left(t^{2}\right)$ it follows that $\Gamma^{\prime}(t)=2 t \alpha^{\prime}\left(t^{2}\right)$ almost everywhere on $[0, \infty)$ and hence $\Gamma^{\prime}(0)$ can be defined as zero. Further, since $\alpha^{\prime}\left(t^{2}\right)$ is bounded on $[0, \epsilon]$ for any $\epsilon>0$ it follows that $\Gamma^{\prime}(t)$ is continuous at $t=0$. Now $\alpha(\cdot)$ is assumed strictly increasing and convex on $[0, \infty)$, so we see $\alpha^{\prime}\left(t^{2}\right)$ is positive almost everywhere there, and furthermore, $\Gamma^{\prime}(t)=2 t \alpha^{\prime}\left(t^{2}\right)$ is strictly increasing on $[0, \infty)$. Let $\psi(s)$ denote a left continuous inverse of $\Gamma^{\prime}(t)=2 t \alpha^{\prime}\left(t^{2}\right)$. Then $\psi(0)=0$ and since $\Gamma^{\prime}(t)$ is continuous at zero and strictly positive for all $t>0$ it follows that $\psi(s)$ is strictly positive for $s>0$. Thus $\Lambda(t)=\int_{0}^{t} \psi(s) d s>0$ for any $t>$ 0 , and hence if $y=\left(y_{1}, y_{2}, \ldots\right)$ is such that $\sum_{i=1}^{\infty} \Lambda\left(\left|y_{i}\right|\right)<\infty$ it follows that $\lim y_{i}=0$. If $W$ denotes all sequences $w$ ith rational coordinates and with only finitely many nonzero coordinates satisfying the condition $\sum_{i=1}^{\infty} \Lambda\left(\left|y_{i}\right|\right) \leq 1$, it follows that $W$ is countable and $\|x\|_{\Gamma}=\sup _{W}\left\{\sum_{i=1}^{\infty}\left|x_{i} y_{i}\right|\right\}$. Hence $\|x\|_{\Gamma}$ is measurable on $H_{\lambda}$ since for each $y \in W$ the function $\sum_{i=1}^{\infty}\left|x_{i}, y_{i}\right|$ is measurable on $H_{\lambda}$.

We will use the symbol $\|\cdot\|_{\Gamma},\|\cdot\|_{\Gamma^{c}},\|\cdot\|_{\lambda},\|\cdot\|_{a},\|\cdot\|_{a_{c}}$ for the norm on $E_{a}, E_{a}^{*}, H_{\lambda}, S_{a}$, and $S_{a_{c}}$, respectively.

Lemma 7.2. Let $F_{n}$ be a sequence of finite Borel measures on $E_{a}$ such that $e\left(F_{n}\right)$ is shift-compact. Then

$$
\sup _{n} \int_{\mathbf{U}}\|x\|_{\boldsymbol{\lambda}}^{2} d F_{n}(x)<\infty
$$

where $\mathcal{U}=\left\{x: \sum_{i=1}^{\infty} \alpha\left(x_{i}^{2}\right) \leq 1\right\}$.

Proof. If $E_{a}=l_{2}, a(t)=t, \lambda=(1,1, \ldots)$ this follows directly from [11, p. 223]. Otherwise, if $x=\left\{x_{i}\right\} \in \mathcal{U}$ then by [10, p. 82] 


$$
\begin{aligned}
\|x\|_{\lambda}^{2} & =\sum_{i=1}^{\infty} \lambda_{i} x_{i}^{2} \leq\|\lambda\|_{a_{c}}\left\|x_{i}^{2}\right\|_{a} \\
& \leq\|\lambda\|_{a_{c}}\left\{1+\sum_{i=1}^{\infty} a\left(x_{i}^{2}\right)\right\} \leq 1
\end{aligned}
$$

since $\sum_{i=1}^{\infty} \alpha\left(x_{i}^{2}\right) \leq 1$ and $\|\lambda\|_{a_{c}} \leq 1 / 2$. Thus $U$ is a measurable subset of the unit ball of $H_{\lambda}$ where the measurability of $\mathcal{U}$ follows since $\sum_{i=1}^{\infty} \alpha\left(x_{i}^{2}\right)$ is a measurable function in $H_{\lambda}$. Now using the arguments employed in the proof of Theorems 6.1 and 6.2 we can show that a sequence of points $\left\{p_{N}\right\}$ in $E_{a}=S_{\Gamma}$ converges to a point $p$ in the norm $\|\cdot\|_{\Gamma}$ iff

$$
\lim _{N} \sum_{i=1}^{\infty} \Gamma\left(\left|x_{N, i}-x_{i}\right|\right)=\lim _{N} \sum_{i=1}^{\infty} \alpha\left(\left|x_{N, i}-x_{i}\right|^{2}\right)=0
$$

where $p_{N}=\left\{x_{N, i}\right\}, p=\left\{x_{i}\right\}$. Further, the right-hand limit in (7.3) equals zero iff the sequence $\left\{q_{N}\right\}$ converges to zero in $S_{\alpha}$ where $q_{N}$ has $i$ th coordinate $\left(x_{i, N}-x_{i}\right)^{2}$. Hence by (7.2), $\lim \left\|p_{N}-p\right\|_{\Gamma}=0$ implies $\lim _{N}\left\|p_{N}-p\right\|_{\lambda}=0$, so $f$ continuous on $H_{\lambda}$ implies $f$ is continuous with respect to the norm $\|\cdot\|_{\Gamma}$ when restricted to $E_{a}$. Thus $e\left(F_{n}\right)$ shift-compact on $E_{a}$ implies it is shift-compact on $H_{\lambda}$ and since the unit ball of $H_{\lambda}$ contains $U$ it follows from $[11$, p. 223] that (7.1) holds.

Lemma 7.3. Suppose $F_{n}$ is an increasing sequence of finite measures on $E_{a}$ such that $e\left(F_{n}\right)$ is shift-compact. Then $F_{n}$ increases to a measure $F$ which is finite on the complement of every neighborbood of zero and such that

$$
J=\sum_{i=1}^{\infty} a\left[\int_{\mathbf{u}} x_{i}^{2} d F(x)\right]<\infty,
$$

and

$$
J=\lim _{n} \sum_{i=1}^{\infty} \alpha\left[\int_{\mathcal{U}_{i}} x_{i}^{2} d F_{n}(x)\right]
$$

where $\mathcal{U}=\left\{x: \sum_{i=1}^{\infty} \alpha\left(x_{i}^{2}\right) \leq 1\right\}$.

Proof. Since $F_{n}$ is increasing we let $F=\lim _{n} F_{n}$. Now $e\left(F_{n}\right)$ shift-compact implies by [11, p. 214] and Remark 2.3 of [11] that $F$ is finite on the complement of every neighborhood of zero in $E_{a}$ and by Lemma 7.2 that

$$
\sum_{i=1}^{\infty} \lambda_{i} \int_{\mathcal{U}} x_{i}^{2} d F_{n}(x)<\infty
$$

If $E_{\alpha}=l_{2}, \lambda=(1,1, \cdots), \alpha(t)=t$ the lemma now follows. In general, since $\lambda=$ $\left\{\lambda_{i}\right\}$ is an arbitrary vector in the positive cone of $S_{a_{c}}$ of norm less than or equal 
to one-hair and $S_{a}$ is the dual of $S_{a_{c}}$ we find that the sequence $\left\{\int_{\mathcal{U}} x_{i}^{2} d F(x)\right\}$ is in $S_{a}$. This implies (7.4), and (7.5) then follows from the dominated convergence theorem since $F_{n}$ increases to $F, \alpha(\cdot)$ is continuous, and

$$
\lim _{n} \int_{\mathcal{U}} x_{i}^{2} d F_{n}(x)=\int_{\mathcal{U}} x_{i}^{2} d F(x)
$$

for $i=1,2, \cdots$.

Lemma 7.4. Let $\mu_{n}=e\left(F_{n}\right)$ where $F_{n}$ is an increasing sequence of measures on $E_{a}$ and suppose the sequence $\mu_{n} * \delta_{x_{n}}$ converges weakly to $\mu$ for some sequence of points $\left\{x_{n}\right\}$ in $E_{a}$. Then $F_{n}$ increases to a measure $F$ as in Lemma 7.3 such that (7.4) and (7.5) hold. Furthermore, the Fourier transform of $\mu$ is

$$
\begin{array}{r}
\phi(y)=\exp \left\{i\left(x_{0}, y\right)+\int_{E_{\alpha}-\mathrm{U}}\left[e^{i(x, y)}-1-\frac{i(x, y)}{1+\|x\|_{\Gamma}^{2}}\right] d F(x)\right. \\
\left.+\int_{\text {บ }}\left[e^{i(x, y)}-1-\frac{i(x, y)}{1+\|x\|_{\lambda}^{2}}\right] d F(x)\right\}
\end{array}
$$

where $\mathcal{U}=\left\{x \in E_{\alpha}: \sum_{i=1}^{\infty} \alpha\left(x_{i}^{2}\right) \leq 1\right\}$.

Proof. Let $\rho_{n}$ be the shift of $\mu_{n}$ by

$$
z_{n}=-\int_{E_{\alpha}-\mathrm{u}} \frac{x}{1+\|x\|_{\Gamma}^{2}} d F_{n}(x)-\int \frac{x}{1+\|x\|_{\lambda}^{2}} d F_{n}(x)
$$

where the integrals are Bochner integrals. Now the function in the first integral in (7.6) is uniformly bounded for any $y$ in a bounded sphere in the dual of $E_{a}$, and by (6.5) $\mathcal{U}$ contains an open neighborhood of zero. Thus $\left\{F_{n}\right\}$ converges weakly to $F$ on $E_{a}-\mathcal{U}[11$, p. 214], and if $S$ is a bounded sphere in the dual of $E_{a}$ then

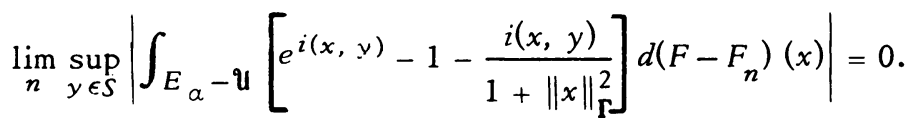

We now consider the second integral in (7.6). Now $\mid e^{i(x, y)}-1-$ $i(x, y) /\left(1+\|x\|_{\lambda}^{2}\right)\left|\leq 1 / 2(x, y)^{2}+\right|(x, y) \mid\|x\|_{\lambda}^{2}$ and $|(x, y)| \leq\|x\|_{\Gamma}\|y\|_{\Gamma} c \leq$ $2\|y\|_{\Gamma}$ since $x \in \mathcal{U}$ which implies $\|x\|_{\Gamma} \leq 2$. Let $K_{n}=F-F_{n}$. Then, for $S$ any bounded sphere in the dual of $E_{a}$, we have

$$
\lim _{n} \sup _{y \in S} \int_{\mathcal{U}}|(x, y)|\|x\|_{\lambda}^{2} d K_{n}(x)=0
$$

since $F_{n}$ increases to $F$ and (7.1) holds in the present setting. Thus to show the second integral in (7.6) is the limit of the corresponding integrals when $F_{n}$ replaces $F$ uniformly for $y$ in a bounded sphere $S$ of the dual of $E_{a}$ we need 
only verify that

$$
\lim _{n} \sup _{y \in S} \int_{\mathcal{U}}(x, y)^{2} d K_{n}(x)=0 .
$$

Let $s_{i j}^{(n)}=\int_{\mathcal{U}^{x}} x_{i} x_{j} d K_{n}(x)$ for $i, j, n=1,2, \ldots$. Then $\left|s_{i j}^{(n)}\right| \leq$ $\int_{\mathcal{U}}\left|x_{i} x_{j}\right| d K_{n}(x) \leq\left[s_{i i}^{(n)}\right]^{1 / 2}\left[s_{j j}^{(n)}\right]^{1 / 2}$ and since $s_{j j}^{(n)} \leq \int_{\mathcal{U}} x_{j}^{2} d F(x)$ it follows from (7.4) that

$$
\sum_{j=k}^{\infty} \alpha\left(s_{j j}^{n}\right) \leq \sum_{j=k}^{\infty} \alpha\left(\int_{\mathrm{u}_{j}} x_{j}^{2} d F(x)\right)<\infty
$$

for $k=1,2, \cdots$. Now for each $y$ in the dual of $E_{\alpha}$ we have $(x, y)^{2}=$ $\sum_{i, j=1}^{\infty} x_{i} x_{j} y_{i} y_{j}$ and since

it follows that

$$
\begin{aligned}
\int_{\text {U }} \sum_{i, j}^{\infty}\left|x_{i} x_{j} y_{i} y_{j}\right| d K_{n}(x) & =\sum_{i, j} \int_{U^{\prime}}\left|x_{i} x_{j} y_{i} y_{j}\right| d K_{n}(x) \\
& \leq\left\{\sum_{i=1}^{\infty}\left[s_{i i}^{(n)}\right]^{1 / 2}\left|y_{i}\right|\right\}^{2}<\infty
\end{aligned}
$$

$$
\int_{\mathcal{U}}(x, y)^{2} d K_{n}(x)=\sum_{i, j=1}^{\infty} s_{i j}^{(n)} y_{i} y_{j}
$$

(7.13) $\quad \int_{\mathcal{U}}(x, y)^{2} d K_{n}(x) \leq\left(\sum_{j=1}^{\infty}\left[s_{j j}^{(n)}\right]^{1 / 2}\left|y_{j}\right|\right)^{2} \leq\left\|\left[s_{j j}^{(n)}\right]^{1 / 2}\right\|_{\Gamma}^{2}\|y\|_{\Gamma^{c}}^{2}$.

Thus (7.10) holds if

$$
\lim _{n}\left\|\left[s_{i j}^{(n)}\right]^{1 / 2}\right\|_{\Gamma}=0
$$

Using (7.3), we see (7.14) holds iff

$$
\lim _{n} \sum_{j=1}^{\infty} a\left(s_{i j}^{(n)}\right)=0
$$

and by (7.11) we have (7.15) if

$$
\lim _{n} s_{j j}^{(n)}=0
$$

Now (7.16) holds since $\lim _{n} \int_{\mathcal{U}} x_{j}^{2} d F_{n}(x)=\int_{\mathcal{U}} x_{j}^{2} d F(x)$, for $j=1,2, \ldots$.

If $F_{n, 1}$ and $F_{n, 2}$ denote $F_{n}$ restricted to $\mathcal{U}$ and $E_{a}-\mathcal{U}$, respectively, we have that

$$
\text { (7.17: } \quad e\left(F_{n}\right) * \delta_{x_{n}}=e\left(F_{n, 1}\right) * e\left(F_{n, 2}\right) * \delta_{z_{n}} * \delta_{x_{n}-z_{n}}
$$

where $z_{n}$ is defined as in (7.7). Now the sequence $\left\{e\left(F_{n}\right) * \delta_{x_{n}}\right\}$ converges weakly to $\mu$ thus (7.17) implies 


$$
\nu_{n}=e\left(F_{n, 1}\right) * e\left(F_{n, 2}\right) * \delta_{z_{n}}
$$

is shift-compact. Using (7.9), (7.9), and (7.10) it follows that the Fourier transforms of the sequence of measures $\left\{\nu_{n}\right\}$ converge uniformly on bounded spheres of the dual of $E_{a}$ to the right-hand side of (7.6) withut the term $\exp \left\{i\left(x_{0}, y\right)\right\}$. Thus $\left\{\nu_{n}\right\}$ actually converges to a measure $\nu$, and hence by (7.17) and the fact that $e\left(F_{n}\right) * \delta_{n}$ converges to $\mu$ the sequence of points $\left\{x_{n}-z_{n}\right\}$ converges to a point $x_{0} \in E_{a}$. Furthermore, we have the Fourier transform of $\mu$ is $\exp \left\{i\left(x_{0}, y\right)\right\}$ multiplied by the Fourier transform of $\nu$ so the lemma is proved.

In $\$ 5$ we said a measure on $E_{\alpha}$ was Gaussian with mean $a \in E_{a}$ if the distribution of every linear functional $x$ in the dual of $E_{a}$ is Gaussian with mean $x(a)$. Varadhan $[11$, p.215] defines a Gaussian measure as any measure $\mu$ which is infinitely divisible and such that if $\mu=e(F) * \gamma$ where $\gamma$ is infinitely divisible then $F$ is degenerate at zero. The definition of infinite divisibility is the usual one. The purpose of our next result is to show that these definitions coincide on $E_{\alpha}$.

Theorem 7.1. If $\mu$ is a measure on $E_{a}$ the following are equivalent:

(i) $\mu$ bas Fourier transform of the form

$$
\phi(x)=\exp \{i\langle x, a\rangle-1 / 2 T(x, x)\}
$$

where $T$ is an a-operator on the dual of $E_{a}$ satisfying

$$
T\left(\beta_{i}, \beta_{j}\right)=\int_{E_{\alpha}} \beta_{i}(y-a) \beta_{j}(y-a) d \mu(y)
$$

for $i, j=1,2, \cdots$ and $a \in E_{a}$.

(ii) For each vector $f$ in the dual of $E_{a}$ the linear functional $f(\cdot)$ on $E_{a}$ bas a Gaussian distribution with mean $f(a)$ for some $a$ in $E_{\alpha}$.

(iii) $\mu$ is infinitely divisible and if $\mu=e(F) * \gamma$ where $\gamma$ is infinitely divis. ible then $F$ is degenerate at zero.

Proof. By Theorem 5.1, Corollary 5.1, Theorem 6.3 and Corollary 6.1 it follows that (i) and (ii) are equivalent. Now assume (ii) holds and let $f$ be any linear functional on $E_{\alpha}$. Now $\mu$ is infinitely divisible since (ii) implies (i) and the class of $\alpha$-operators is closed under multiplication by constants. Thus if $\mu=e(F) * \gamma$ where $\gamma$ is infinitely divisible we must show $F$ is degenerate at zero. Now an easy computation shows that the measure $\mu^{f}(A)=\mu\left(f^{-1}(A)\right)$ defined on the real line satisfies

$$
\mu^{f}=e\left(F^{f}\right) * \gamma^{\prime}
$$

where $F^{f}$ and $\gamma^{f}$ are defined in the same manner as $\mu^{f}$. Similarly, $\gamma$ being 
infinitely divisible implies that $\gamma^{f}$ is infinitely divisible, and since $\mu^{f}$ is Gaussian on the real line we then have $F^{f}$ degenerate at zero. Thus for each continuous linear functional $f$ on $E_{a}$ the measure $F f$ is degenerate at zero and this implies $F$ itself is concentrated at the origin in $E_{\alpha}$ which proves that (ii) implies (iii).

Now assume (iii) holds and let $H_{\lambda}$ be defined as in Lemma 7.1. Then $\mu$ is infinitely divisible on $H_{\lambda}$ and if $\mu=e(F) * \gamma$ where $\gamma$ is infinitely divisible on $H_{\lambda}$ and $F$ is some finite measure on $H_{\lambda}$ it follows that $F$ is degenerate at zero. That is, if $\mu$ is a probability measure on $H_{\lambda}$ with $\mu\left(E_{a}\right)=1$ and $\mu=e(F) * \gamma$ where $F$ is some finite measure on $H_{\lambda}$ and $\gamma$ is a probability measure on $H_{\lambda}$ then $F$ and $\gamma$ are concentrated on $E_{\alpha}$. Hence (iii) holds when $\mu$ is viewed as a measure on $H_{\lambda}$ and by $\left[11\right.$, p. 226] every linear functional $f$ on $H_{\lambda}$ has a Gaussian distribution. Now $f$ restricted to $E_{\alpha}$ is continuous and since $E_{\alpha}$ is dense in $H_{\lambda}$ the restriction maps $H_{\lambda}$ into the dual of $E_{a}$ in one-to-one fashion. By Lemma 2.1 it then follows that each $f$ in the dual of $E_{\alpha}$ is the weak-star limit of restrictions to $E_{a}$ of elements in the dual of $H_{\lambda}$, and since limits of Gaussian distributions are Gaussian (possibly degenerate) it follows that each linear functional on $E_{a}$ has a Gaussian distribution. In view of Corollary 5.1 it follows that (iii) implies (ii).

Theorem 7.2. Let $\mu$ be a measure on $E_{\alpha}$. Then $\mu$ is infinitely divisible on $E_{a}$ iff the Fourier transform of $\mu$ is of the form

$$
\begin{aligned}
\phi(y, \mu)=\exp \left\{i\left(x_{0}, y\right)-\frac{1}{2} T(y, y)+\int_{E_{a}-\mathcal{U}}\left[e^{i(x, y)}-1-\frac{i(x, y)}{1+\|x\|_{\Gamma}^{2}}\right] d F(x)\right. \\
\left.+\int_{\mathcal{U}}\left[e^{i(x, y)}-1-\frac{i(x, y)}{1+\|x\|_{\lambda}^{2}}\right] d F(x)\right\}
\end{aligned}
$$

where $x_{0} \in E_{\alpha}, T$ is an a-operator, $\mathcal{U}=\left\{x \in E_{a}: \sum_{i=1}^{\infty} \alpha\left(x_{i}^{2}\right) \leq 1\right\}$, and $F$ is a $\sigma$-finite measure on $E_{\alpha}$ with finite mass outside every neighborbood of zero and no mass at zero satisfying

$$
\sum_{i=1}^{\infty} \alpha\left[\int_{\mathcal{U}} x_{i}^{2} d F(x)\right]<\infty .
$$

For each fixed $\lambda$ the representation is unique.

Proof. First we assume $\mu$ has Fourier transform (7.19) and $F$ satisfies (7.20). We now observe that $\mu=g * \nu$ where $g$ and $\nu$ are measures on $E_{a}$ with Fourier transforms 


$$
\begin{aligned}
& \hat{g}(y)=\exp \left\{i\left(x_{0}, y\right)-\frac{1}{2} T(y, y)+\int_{E_{\alpha}-\mathcal{U}}\left[e^{i(x, y)}-1-\frac{i(x, y)}{1+\|x\|_{\Gamma}^{2}}\right] d F(x)\right\} \\
& \hat{v}(y)=\exp \left\{\int_{\mathcal{U}}\left[e^{i(x, y)}-1-\frac{i(x, y)}{1+\|x\|_{\lambda}^{2}}\right] d F(x)\right\}
\end{aligned}
$$

That the measure $g$ exists follows immediately from Theorem 5.2 and that $F$ restricted to $E_{a}-\mathcal{U}$ is finite. Furthermore, it is obvious that $g$ is infinitely divisible. The existence of $\nu$ proceeds as follows. From (7.20) it follows that $\int_{\mathcal{U}}\|x\|_{\lambda}^{2} d F(x)<\infty$, and hence if $F$ restricted to $\mathcal{U}$ is viewed as a measure on $H_{\lambda}$ we have the existence of the measure $\nu$ on $H_{\lambda}[11$, p. 225] with Fourier transform as in (7.22). Furthermore, $\nu$ is infinitely divisible on $H_{\lambda}$ and since $\mu=g * \nu$ where $\mu$ and $g$ assign mass one to $E_{a}$ it follows that $\nu\left(E_{\alpha}\right)=1$ and that $\nu$ is infinitely divisible on $E_{a}$. Thus both $g$ and $\nu$ exist on $E_{a}$ and they are infinitely divisible so $\mu$ is infinitely divisible.

Now we assume $\mu$ is infinitely divisible on $E_{a}$. To prove $\mu$ has a Fourier transform of the form (7.19) where $F$ satisfies (7.20) we can proceed exactly as in [11]. That is, we choose a sequence of probability measures $e\left(F_{n}\right)$ where $F_{n}$ increases to, say, $F, e\left(F_{n}\right) * \delta_{x_{n}}$ converges to a measure $\lambda$, and $\mu=\lambda * g$ where $g$ is Gaussian. Then by Lemma 7.4 and Theorem 7.1 the Fourier transform of $\mu$ is of the form (7.19) and, by Lemma 7.3, F satisfies (7.20) along with the other conditions of the theorem. The uniqueness follows exactly as indicated in [11].

The following corollary relates to the results found in [11].

Corollary 7.1. A function $\phi(y)$ is the Fourier transform of an infinitely divisible probability measure $\mu$ on $l_{2}$ iff

$$
\phi(y)=\exp \left\{i\left(x_{0}, y\right)-\frac{1}{2}(T y, y)+\int_{l_{2}} K(x, y) d F(x)\right\}
$$

where $x_{0} \in l_{2}, K(x, y)=e^{i(x, y)}-1-i(x, y) /\left(1+\|x\|^{2}\right), T$ is an S-operator on $l_{2}$, and $F$ is a $\sigma$-finite measure on $l_{2}$ with finite mass outs ide every neighborbood of zero and satisfying $\int_{B}\|x\|^{2} d F(x)<\infty$ where $B=\left\{x \in l_{2}:\|x\| \leq 1\right\}$.

Proof. In view of Theorems 7.1, 7.2, Corollary 5.3, and Corollary 3.1 it suffices to prove that $\gamma(y)=\exp \left\{\int_{l_{2}} K(x, y) d F(x)\right\}$ is continuous in the $\tau_{2}$-topology since it follows easily that $\gamma(0)=1, \gamma$ is positive definite, and that any family of measures on $l_{2}$ is a $\lambda$-family with $\lambda=(1,1, \cdots)$. Further, since $F$ is finite on $l_{2}-B$ it follows that $\exp \left\{\int_{l_{2}-B} K(x, y) d F(x)\right\}$ is the Fourier transform of a probability measure on $l_{2}$ and hence it is $\tau_{2}$-continuous. Thus we need only show 


$$
\exp \left\{\int_{B} K(x, . y) d F(x)\right\}
$$

is $\tau_{2}$-continuous. Now proceeding as in Lemma 7.4,

$$
\begin{aligned}
& \left|\int_{B} K(x, y) d F(x)\right| \\
& \quad \leq \frac{1}{2} \int_{B}(x, y)^{2} d F(x)+\left[\int_{B}(x, y)^{2} d F(x)\right]^{1 / 2} \cdot\left\{\int_{B}\|x\|^{4} d F(x)\right\}^{1 / 2}
\end{aligned}
$$

and, since $\int_{B}(x, y)^{2} d F(x)$ is an $S$-operator on $l_{2},(7.25)$ implies (7.24) is $\tau_{2}$ continuous. This proves $\gamma(y)$ is $\tau_{2}$-continuous and completes the proof.

8. Infinitely divisible distributions as limiting distributions. We now consider the derivation of the limiting distribution of the sum of independent random variables taking values in the Orlicz space $E_{a}$ as defined in $\$ 7$. For each inte ger $n$, we are given $k_{n}$ random variables which are mutually independent. We shall denote these by $X_{n j}$ where $j=1, \cdots, k_{n}$ and letting $Y_{n}=\sum_{j=1}^{k_{n}} X_{n j}$ our purpose is to derive the limiting distribution of $Y_{n}$ and $Y_{n}$ suitably shifted. We need the following definition.

Let $\left\{X_{n j}\right\}$ be a sequence of random variables taking values in $E_{a}$ and.let the sequence of probability measures induced on $E_{a}$ be denoted by $\left\{F_{n j}\right\}$. Then $\left\{X_{n j}\right\}$, or equivalently $\left\{F_{n j}\right\}$, is said to be uniformly infinitesimal if for every $\epsilon>0$

$$
\lim _{n} \sup _{1 \leq j \leq k_{n}} F_{n j}\left(\left\{x \in E_{a}:\|x\|_{\Gamma}^{\prime}>\epsilon\right\}\right)=0 .
$$

The following theorem on Poisson sums of random variables in a separable Banach space is due to Lucien LeCam [13].

Theorem 8.1. Let $X_{n j i}\left(j=1, \cdots, k_{n} ; n, i=1,2, \cdots\right)$ be independent random variables with values in a separable Banach space with the distribution of $X_{n j i}$ being $F_{n j}$ for $i=1,2, \ldots$. Let $\left\{N_{n j}\right\}$ be Poisson random variables independent among themselves as well as independent of all the $X_{n j i}$ and each with parameter one. Let $G_{n}$ denote the distribution of

$$
T_{n}=\sum_{j=1}^{k_{n}} \sum_{i=1}^{N_{n j}} X_{n j i}
$$

If $G_{n}$ is condition ally compact then $\Pi_{j=1}^{k_{n}} F_{n j}$ is shift-compact where $\Pi_{j=1}^{k_{n}} F_{n j}$ denotes the convolution of the indicated measures.

We now fix some notation to be used throughout the remainder of the paper. We let $B=\left\{x \in E_{a}:\|x\|_{\Gamma} \leq \rho\right\}$ where $0<\rho \leq 1$ and $B \subseteq \mathcal{U}=\left\{x: \sum_{i=1}^{\infty} \alpha\left(x_{i}^{2}\right) \leq 1\right\}$. The existence of such a $\rho$ follows from the arguments of Theorems 6.1 and 6.3 and the definition of the quasi-norm of Theorem 6.1. Additional notation we will use is 


$$
\begin{array}{lll}
\mu_{n}=\prod_{j=1}^{k} F_{n j}, & x_{n j}=\int_{B} x d F_{n j}(x), & \beta_{n j}=F_{n j}^{*}\left(\delta_{-x}\right), \\
x_{n}=\sum_{j=1}^{k} x_{n j}, & F_{n}=\sum_{j=1}^{k} \beta_{n j}, & \lambda_{n}=e\left(F_{n}\right) * \delta_{x_{n}} .
\end{array}
$$

Lemma 8.1. Let $F_{n j}\left(j=1, \cdots, k_{n} ; n=1,2, \cdots\right)$ be a uniformly infinites. imal sequence of probability.measures on $E_{a}$ and assume the notation in (8.1). Then

(i) $\lim _{n} \max _{1 \leq j \leq k_{n}}\left\|x_{n j}\right\|_{\Gamma}=0$,

(ii) there exists an $N$ such that for all $1 \leq j \leq k_{n}$ and $n \geq N^{+}$

$$
\left\|\int_{B} x d \beta_{n j}(x)\right\|_{\Gamma} \leq 2 \beta_{n j}\left\{x:\|x\|_{\Gamma}>\rho / 2\right\} \text {. }
$$

Proof. Take $\epsilon>0$ and let $V=\left\{x:\|x\|_{\Gamma}<\epsilon\right\}$. Then

$$
\left\|x_{n j}\right\|_{\Gamma} \leq \int_{V}\|x\|_{\Gamma} d F_{n j}(x)+F_{n j}\left\{x: \epsilon<\|x\|_{\Gamma} \leq \rho\right\} \leq \epsilon+F_{n j}\left(E_{\alpha}-V\right) .
$$

and $\left\{F_{n j}\right\}$ being uniformly infinitesimal implies

$$
\lim _{n} \max _{1 \leq j \leq k_{n}}\left\|x_{n j}\right\|_{\Gamma}<\epsilon
$$

so (i) holds. To prove (ii) we first take $N$ such that $\max _{1 \leq j \leq k_{n}}\left\|x_{n j}\right\|_{\Gamma}<\dot{\rho} / 4$ for all $n \geq N$. Then for $n \geq N$ and $1 \leq j \leq k_{n}$,

$$
\begin{aligned}
& \left\|\int_{B} x d \beta_{n j}(x)\right\|_{\mathbf{r}}=\left\|\int_{\left\|_{x-x_{n j}}\right\|_{\mathbf{\Gamma}} \leq \rho}\left(x-x_{n j}\right) d F_{n j}(x)\right\|_{\mathbf{r}} \\
& =\left\|\int_{\left\|x-x_{n j}\right\|_{\Gamma} \leq \rho} x d F_{n j}(x)-x_{n j} F_{n j}\left\{x:\left\|x-x_{n j}\right\| \leq \rho\right\}\right\|_{\Gamma} \\
& =\left\|\int_{\| x-x}\right\|_{\Gamma} \leq \rho \leq F_{n j}(x)-\int_{B} x d F_{n j}(x)+x_{n j} F_{n j}\left\{x:\left\|x-x_{n j}\right\|>\rho\right\} \| \mathbf{\Gamma} \\
& \leq \int_{3 \rho / 4<\|x\|_{\Gamma}<5 \rho / 4}\|x\|_{\Gamma} d F_{n j}(x)+(\rho / 4) \beta_{n j}\left\{x:\|x\|_{\Gamma}>\rho\right\} \\
& \leq(\rho / 4)\left[5 F_{n j}\left\{x:\|x\|_{\Gamma}>3 \rho / 4\right\}+\beta_{n j}\left\{x:\|x\|_{\Gamma}>\rho\right\}\right] \\
& \leq 2 \beta_{n j}\left\{x:\|x\|_{\Gamma}>\rho / 2\right\}
\end{aligned}
$$

so (ii) holds since $\rho \leq 1$. 
Theorem 8.2. Let $\left\{F_{n j}\right\}(j=1, \cdots, k ; n=1,2, \cdots)$ be a uniformly infinitesimal sequence of probability measures on $E_{a}$ and assume the notation in (8.1). Then, if $\left\{\lambda_{n}\right\}$ is conditionally compact we bave $\left\{\mu_{n}\right\}$ shift-compact, and for each uniformly bounded set $S$ in the dual of $E_{a}$

$$
\lim _{n \rightarrow \infty} \sup _{y \in S}\left|\phi\left(y, \lambda_{n}\right)-\phi\left(y, \mu_{n}\right)\right|=0
$$

Proof. If $\left\{\lambda_{n}\right\}$ is conditionally compact then Theorem 8.1 implies $\Pi_{j=1}^{k} \beta_{n j}=$ $\Pi_{j=1}^{k} F_{n j} * \delta_{x_{n}}$ is shift-compact and hence $\mu_{n}=\Pi_{j=1}^{k} F_{n j}$ is shift-compact. To complete our proof we now need to establish (8.2). First we observe that since $\left\{F_{n j}\right\}$ is uniformly infinitesimal there exists an $N$ such that $n \geq N$ implies

$$
\sup _{y \in S} \max _{1 \leq j \leq k}\left|\phi\left(y, \beta_{n j}\right)-1\right|<1 / 2 .
$$

Then, since $|\log (1+z)-z| \leq 2|z|^{2}$ for $|z| \leq^{1 / 2}$ we have

$$
\begin{aligned}
\left|\log \phi\left(y, \lambda_{n}\right)-\log \phi\left(y, \mu_{n}\right)\right| \\
\quad=\left|\sum_{j=1}^{k}\left[\phi\left(y, \beta_{n j}\right)-1\right]+i\left(y, x_{n}\right)-\sum_{j=1}^{k} \log \phi\left(y, \beta_{n j}\right)-i\left(y, x_{n}\right)\right| \\
\quad=\left|\sum_{j=1}^{k}\left[\phi\left(y, \beta_{n j}\right)-1-\log \phi\left(y, \beta_{n j}\right)\right]\right| \\
\leq 2 \sum_{j=1}^{k}\left|\phi\left(y, \beta_{n j}\right)-1\right|^{2} \\
\leq 2 \max _{1 \leq j \leq k_{n}}\left|\phi\left(y, \beta_{n j}\right)-1\right| \sum_{j=1}^{k}\left|\phi\left(y, \beta_{n j}\right)-1\right| .
\end{aligned}
$$

Thus, by (8.4),

$$
\lim _{n \in S} \sup _{y \in S}\left|\log \phi\left(y, \lambda_{n}\right)-\log \phi\left(y, \mu_{n}\right)\right|=0
$$

since $\left\{F_{n j}\right\}$ uniformly infinitesimal implies

$$
\lim _{n} \sup _{y \in S} \max _{1 \leq j \leq k_{n}}\left|\phi\left(y, \beta_{n j}\right)-1\right|=0
$$

and we also have $\overline{\lim }_{n} \sup _{y \in S} \sum_{j=1}^{k_{n}}\left|\phi\left(y, \beta_{n j}\right)-1\right|<\infty$. To see this last assertion notice that 


$$
\begin{aligned}
& \sum_{j=1}^{k}\left|\phi\left(y, \beta_{n j}\right)-1\right|=\sum_{j=1}^{k}\left|\int_{E_{a}}\left[e^{i(x, y)}-1\right] d \beta_{n j}(x)\right| \\
& =\sum_{j=1}^{k} \mid \int_{B}\left[e^{i(x, y)}-1-i(x, y)\right] d \beta_{n j}(x)+i \int_{B}(x, y) d \beta_{n j}(x) \\
& \quad+\int_{E_{a}-B}\left[e^{i(x, y)}-1\right] d \beta_{n j}(x) \mid \\
& \leq \sum_{j=1}^{k}\left[\int_{B}(x, y)^{2} d \beta_{n j}(x)+\|y\|_{E_{\alpha}^{*}}\left\|\int_{B} x d \beta_{n j}(x)\right\| \|_{\Gamma}+2 \beta_{n j}\left(E_{a}-B\right)\right] \\
& \leq \int_{B}(x, y)^{2} d F_{n}(x)+2\left[\|y\|_{\left.E_{a}^{*}+1\right] F_{n}\left\{x:\|x\|_{\Gamma}>\rho / 2\right\} .}\right.
\end{aligned}
$$

Since $e\left(F_{n}\right)$ is conditionally compact this implies $e\left(\sum_{1}^{k_{n}}\left|\beta_{n j}\right|^{2}\right)$ is conditionally compact where $\left|\beta_{n j}\right|^{2}=\beta_{n j} * \bar{\beta}_{n j}$ and $\bar{\beta}_{n j}(A)=\beta_{n j}(-A)$ for all Borel sets $A$. Therefore we obtain from the symmetry of $\left|\beta_{n j}\right|^{2}$ that

$$
\varlimsup_{n} \sup _{y \in S} \sum_{1}^{k} \int_{\|x\|_{\Gamma} \leq 2 \rho}(x, y)^{2}\left|\beta_{n j}\right|^{2}(d x)<\infty .
$$

Hence from conditional compactness of $e\left(F_{n}\right)$ we obtain by an argument similar to $[11$, p. 235$]$ and Lemma 8.1 (ii) that

$$
\varlimsup_{n} \sup _{y \in S} \int_{B}(x, y)^{2} F_{n}(d x)<\infty ;
$$

and further, by $[11$, p. 214$]$ that $\sup _{n} F_{n}\left\{x \mid\|x\|_{\Gamma}>\rho / 2\right\}<\infty$, so that (8.6) implies $\overline{\lim }_{n} \sup _{y \in S} \sum_{1}^{k_{n}}\left|\phi\left(y, \beta_{n j}\right)-1\right|$ is finite. Thus (8.5) holds and this implies (8.2), so the theorem is proved.

Corollary 8.1. Let $\left\{F_{n j}\right\}$ be uniformly infinitesimal. Then, if $\left\{\mu_{n}\right\}$ converges weakly to $\mu$ and $\left\{x_{n}\right\}$ is a compact set in $E_{a}$ we bave $\mu$ infinitely divisible.

Proof. By The orem 8.2 we have $\lim _{n} \phi\left(y, \lambda_{n}\right)=\lim _{n} \phi\left(y, \mu_{n}\right)=\phi(y, \mu)$ for each $y \in E_{a}^{*}$, and by [11, Theorem 7.6$]\left\{\lambda_{n}\right\}$ converges weakly to a measure $\nu$ on $H_{\lambda}$. Since $\phi(y, \nu)=\phi(y, \mu)$ on $H_{\lambda}$ it follows that $\mu=\nu$ on $H_{\lambda}$, and since $\mu\left(E_{\alpha}\right)=$ 1 we have $\nu\left(E_{a}\right)=1$. Further, $\nu$ is infinitely divisible on $H_{\lambda}$ by [11, Theorem 5.1] since each $\lambda_{n}$ is infinitely divisible there, and hence $\mu=\nu$ is infinitely divisible on $E_{a}$. 
9. Convergence of certain infinitely divisible laws and a central limit theorem. We first prove a theorem regarding the convergence of certain infinitely divisible laws on the Orlicz space $E_{a}$ as defined in $\$ 7$. The notation $\mu=$ $\left[x_{0}, T, F\right]$ is used to denote the fact that $\mu$ is infinitely divisible with Fourier transform as in (7.19) having parameters $x_{0}, T, F$ with respect to some fixed $\lambda$.

Theorem 9.1. Let $\mu_{n}=\left[x_{n}, T_{n}, F_{n}\right]$ with respect to some fixed $\lambda$ and assume $\left\{x_{n}\right\}$ is conditionally compact with $F_{n}$ an increasing sequence of finite measures on $E_{a}$. Then $\left\{\mu_{n}\right\}$ is conditionally compact iff

(i) $F_{n}$ restricted to the complement of a neighborbood of zero is weakly conditionally compact,

(ii) the sequence of bilinear forms

$$
R_{n}(y, y)=\int_{\mathcal{U}}(x, y)^{2} d F_{n}(x)+T_{n}(y, y)
$$

satisfies

$$
\begin{array}{r}
\sup _{n} \sum_{i=1}^{\infty} \alpha\left(r_{i i}^{(n)}\right)<\infty, \\
\lim \sup _{n} \sum_{i=N}^{\infty} \alpha\left(r_{i i}^{(n)}\right)=0
\end{array}
$$

where $r_{i i}^{(n)}=R_{n}\left(e_{i}, e_{i}\right), e_{i}$ is the sequence with one in the ith position and zero elsewbere, and $\mathcal{U}=\left\{x \in E_{a}: \sum_{i=1}^{\infty} \alpha\left(x_{i}^{2}\right) \leq 1\right\}$,

(iii) $\left\{\mu_{n}\right\}$ is a $\lambda$-family with respect to the fixed $\lambda$ yielding the representations $\mu_{n}=\left[x_{n}, T_{n}, F_{n}\right]$.

Proof. First assume $\left\{\mu_{n}\right\}$ is conditionally compact. Then $\left\{x_{n}\right\}$ conditionally compact implies $\left\{e\left(F_{n}\right)\right\}$ shift-compact, so (i) follows from [11, p. 2141 and Remark 2.3 of [11]. Since $e_{i}$ represents the coefficient functional $\beta_{i}$ scaled by a fixed positive constant for $i=1,2, \cdots$, we have by Theorem $5.2(3)$, that (9.2) and (9.3) hold with $r_{i i}^{(n)}$ replaced by $t_{i i}^{(n)}=T_{n}\left(e_{i}, e_{i}\right)$. That is, we know the measures $\left[x_{n}, T_{n}, 0\right]$ are shift-compact, thus $\left[0, T_{n}, 0\right]$ is compact, so Theorem 5.2 applies, and the scale factor can be ignored since $\alpha(\cdot)$ satisfies (6.1).

Furthermore, if

$$
s_{i i}^{(n)}=\int_{\mathcal{U}}\left(x, e_{i}\right)^{2} d F_{n}(x) \quad(n, i=1,2, \ldots)
$$

then

$$
s_{i i}^{(n)}=\int_{u} x_{i}^{2} d F_{n}(x)
$$

and letting $F=\lim F_{n}$ as in Lemma 7.3 we have by (7.5) that (9.2) and (9.3) hold 
with $r_{i i}^{(n)}$ replaced by $s_{i i}^{(n)}$. Now $r_{i i}^{(n)}=s_{i i}^{(n)}+t_{i i}^{(n)}$ and since $\alpha(\cdot)$ satisfies (6.1) we have by (6.3) that (9.2) and (9.3) hold as stated. Finally, (iii) holds by Lemma 3.1. Now we assume (i), (ii), and (iii) hold. Now $\mathcal{U}$ contains an open neighborhood $V$ of zero and we can assume $F_{n}$ vanishes on the complement of $V$. The last remark follows from (i) since on $E_{a}-V$ we have $F_{n}$ converging to some finite measure, call it $F$, and hence if $G_{n}$ is $F_{n}$ restricted to $E_{a}-V$ we have $e\left(G_{n}\right)$ converging to $e(F)$. Since the unit ball, call it $W$, of $H_{\lambda}$ contains $\mathcal{U}$ and each $F_{n}$ is concentrated on $V \subseteq \mathcal{U} \subseteq W$ it follows from (ii) that $R_{n}$ is an a-operator. Hence Lemma 5.4 implies that $\exp \left\{-1 / 2 R_{n}(y, y)\right\}$ is the Fourier transform of a Gausșian measure, say $\lambda_{n}$, on $E_{a} \subseteq H_{\lambda}$. It follows from (ii) that $\left\{\lambda_{n}\right\}$ is conditionally compact on $E_{a}$ and hence on $H_{\lambda}$, and hence that the operators $A_{n}$ induced by $R_{n}$ through $\lambda_{n}$ on $H_{\lambda}^{*}$ satisfy the conditions of The orem 6.3 (see $\left[11, \mathrm{p}\right.$. 230]) so $\left\{\mu_{n}\right\}$ is conditionally compact on $H_{\lambda}$. Then us ing (iii) we have $\left\{\mu_{n}\right\}$ conditionally compact on $E_{a}$ so the theorem is proved.

We now proceed to a central limit theorem.

Theorem 9.2. Let $\left\{F_{n j}\right\}\left(j=1, \cdots, k_{n} ; n=1,2, \ldots\right)$ be a uniformly infinitesimal sequence of probability measures on $E_{a}$ and assume the notation in (8.1). Further, assume $\lim _{n} x_{n}=x_{0}$ and that

$$
\begin{array}{r}
\sup _{n} \sum_{i=1}^{\infty} \alpha\left(\int_{\mathcal{U}} x_{i}^{2} d F_{n}(x)\right)<\infty, \\
\lim _{N} \sup _{n} \sum_{i=N}^{\infty} \alpha\left(\int_{\mathcal{U}} x_{i}^{2} d F_{n}(x)\right)=0 .
\end{array}
$$

Then $\Pi_{j=1}^{k} F_{n j}=\mu_{n}$ converges weakly to the Gaussian measure $\mu_{0}=\left[x_{0}, T_{0}, 0\right]$ where $T_{0}$ is an a-operator if for every $\epsilon>0$

(a) $\lim _{n} \sum_{j=1}^{k_{n}} F_{n j}\left\{x:\left\|x-x_{n j}\right\|_{\Gamma}>\epsilon\right\}=0$,

(b) $\lim _{n} \sum_{j=1}^{k_{n}} \int_{\|x\|_{\Gamma} \leq \epsilon}\left(x-x_{n j}, y\right)^{2} d F_{n j}(x)=T_{0}(y, y)$ for eacb $y \in E_{a}^{*}$,

(c) $e\left(F_{n}\right)$ is a $\lambda$-family.

Proof. Now (a) implies $\lim _{n} F_{n}\left(E_{\alpha}-V\right)=0$ for every open neighborhood $V$ of zero, and hence if $G_{n}$ is $F_{n}$ restricted to $E_{a}-V$ we have $e\left(G_{n}\right)$ converging to the unit mass at zero. Now let $H_{n}$ be $F_{n}$ restricted to $V$ where $V=\left\{x \in E_{a}\right.$ : $\left.\|x\|_{\Gamma}<y\right\} \subseteq B$. Then $e\left(H_{n}\right)=\left[z_{n}, 0, H_{n}\right]$ for any $\lambda$ where

$$
z_{n}=\int_{U_{1}} \frac{x}{1+\|x\|_{\lambda}^{2}} d H_{n}(x)=\int_{V} \frac{x}{1+\|x\|_{\lambda}^{2}} d F_{n}(x) .
$$

Now let $\epsilon>0$ be given. Then 


$$
\begin{aligned}
z_{n} & =\int_{V} x d F_{n}(x)-\int_{V} \frac{x\|x\|_{\lambda}^{2}}{1+\|x\|_{\lambda}^{2}} d F_{n}(x) \\
& =x_{n}-\int_{B-V} x d F_{n}(x)-\int_{V} \frac{x\|x\|_{\lambda}^{2}}{1+\|x\|_{\lambda}^{2}} d F_{n}(x)
\end{aligned}
$$

and since

$$
\begin{gathered}
\left\|\int_{B-V} x d F_{n}(x)\right\|_{\Gamma} \leq \rho F_{n}\{B-V\}, \\
\left\|\int_{V} \frac{x\|x\|_{\lambda}^{2}}{1+\|x\|_{\lambda}^{2}} d F_{n}(x)\right\|_{\Gamma} \leq \epsilon \int_{\|x\|_{\Gamma} \leq \epsilon}\|x\|_{\lambda}^{2} d F_{n}(x)+\gamma F_{n}\left\{x: \epsilon<\|x\|_{\Gamma}<\gamma\right\},
\end{gathered}
$$

we have $\overline{\lim }_{n}\left\|z_{n}-x_{n}\right\|_{\Gamma} \leq \epsilon$ by applying (a) and (9.5). Thus $\lim z_{n}=\lim x_{n}=x_{0}$ and by (9.5), (9.6), and (a) we have by the reasoning used in Theorem 9.1 that $e\left(F_{n}\right)$ is conditionally compact on $H_{\lambda}$. Then by $(c)$ it follows that $e\left(F_{n}\right)$ is conditionally compact on $E_{a}$. Further, in view of (a), (b) each linear functional has a limiting Gaussian distribution with mean zero and variance $T_{0}(y, y)$ with respect to all limits of $e\left(F_{n}\right)$. Hence $e\left(F_{n}\right)$ converges to $\gamma=\left[0, T_{0}, 0\right]$ and by Theorem 8.2 we then have $\prod_{j=1}^{k} F_{n j}$ converging weakly to $\gamma$ so the theorem is proved.

Corollary 9.1. Let $\left\{F_{n j}\right\}$ be a uniformly infinitesimal sequence of probability measures on $E_{a}$ and assume the notation of (8.3). Further, assume $\lim x_{n}=x_{0}$ and that there exists a measure $\rho$ on $E_{a}$ such that

$$
\begin{aligned}
& \sum_{i=1}^{\infty} a\left(\int_{E_{a}} x_{i}^{2} d \rho(x)\right)<\infty, \\
& \int_{\mathcal{U}} x_{i}^{2} d F_{n}(x) \leq \int_{E_{a}} x_{i}^{2} d \rho(x) \quad(i=1,2, \ldots) .
\end{aligned}
$$

Then conditions (a), (b), (c) of Theorem 9.2 imply that $\Pi_{j}^{k}=_{1} F_{n j}$ converge weakly to $\mu_{0}=\left[x_{0}, T_{0}\right.$, ol.

Proof. We need only show (9.5) and (9.6) hold and that is obvious by (9.9) and (9.10).

Corollary 9.2. Let $\mu$ be a probability measure on $E_{a}$ such that (9.9) bolds for $\mu, \int_{E_{\alpha}}\|x\|_{\Gamma}^{2} d \mu(x)$ is finite, and $\int_{E_{\alpha}} x d \mu(x)=0$. Let $Z_{1}^{a}, Z_{2}, \cdots$ be independent 
random variables such that $\mathfrak{Q}\left(Z_{k}\right)=\mu$ for $k=1,2, \ldots$ and define $S_{n}=Z_{1}+$ $\cdots+Z_{n}$. Then $\mathfrak{L}\left(S_{n} / \sqrt{n}\right)$ converges weakly to the Gaussian measure $\mu_{0}=$ $\left[0, T_{0}, 0\right]$, where

$$
T_{0}(y, y)=\int_{E_{a}}(x, y)^{2} d \mu(x)
$$

provided the measures $\gamma_{n}=\mathfrak{L}\left(S_{n} / \sqrt{n}\right)$ or the measures $e\left(F_{n}\right)$ form a $\lambda$-family. Here $F_{n}$ is as in (8.3) with $F_{n j}=\mathscr{\varrho}\left(Z_{j} / \sqrt{n}\right)$ for $j=1, \cdots, n ; n=1,2, \cdots$.

Proof. Since (9.9) holds and $\int_{E_{\alpha}}\|x\|_{\Gamma}^{2} d_{\mu}(x)$ is finite it follows that $T_{0}(y, y)$ is an $\alpha$-operator and hence the Gaussian measure $\mu_{0}=\left[0, T_{0}, 0\right]$ exists on $E_{a} \subseteq H_{\lambda}$. Further, since the $H_{\lambda}$ topology on $E_{\alpha}$ is weaker than the $E_{a}$ topology there exists a constant $c>0$ such that $c\|x\|_{\lambda} \leq\|x\|_{\Gamma}$ for each $x \in E_{\alpha}$ and hence

$$
\int_{H_{\lambda}}\|x\|_{\lambda}^{2} d \mu(x)=\int_{E_{a}}\|x\|_{\lambda}^{2} d \mu(x) \leq\left[\frac{1}{c}\right]^{2} \int_{E_{a}}\|x\|_{\Gamma}^{2} d \mu(x)<\infty .
$$

Thus $\gamma_{n}$ converges weakly to $\mu_{0}$ on $H_{\lambda}\left[9\right.$, p. 1561] and in the case $\gamma_{n}$ is a $\lambda$ family we then have $\gamma_{n}$ converging to $\mu_{0}$ on $E_{a}$. In the case $e\left(F_{n}\right)$ is a $\lambda$ family we apply Corollary 9.1. To verify the hypothesis of Corollary 9.1 we first observe that $\lim _{n} x_{n}=0$. To see this notice that

$$
x_{n}=n \int_{B} x d F_{n j}(x)=\sqrt{n} \int_{\sqrt{n} B} x d \mu(x)
$$

and since $\int_{B} x d \mu(x)=0$ we have

$$
\left\|x_{n}\right\|_{\Gamma}=\left\|-\sqrt{n} \int_{E_{\alpha}-\sqrt{n B}} x d \mu(x)\right\|_{\Gamma} \leq \frac{1}{\sqrt{n}} \int_{E_{a}}\|x\|_{\Gamma}^{2} d \mu(x) .
$$

Further, for every $\epsilon>0$,

$$
\begin{aligned}
\lim _{n} \sum_{j=1}^{n} F_{n j}\{x: & \left.\left\|x-x_{n j}\right\|_{\Gamma}>\epsilon\right\}=\lim _{n} n \mu\left\{x:\left\|x-x_{n j}\right\|_{\Gamma}>\sqrt{n} \epsilon\right\} \\
& \leq \lim _{n} \frac{1}{\epsilon^{2}} \int_{\left\{x:\left\|x-x_{n j}\right\|_{\Gamma}>\sqrt{n} \epsilon\right\}}\left\|x-x_{n j}\right\|_{\Gamma}^{2} d \mu(x)=0
\end{aligned}
$$

since $\int_{E_{\alpha}}\|x\|_{\Gamma}^{2} d \mu(x)$ is finite and $\lim _{n}\left\|x_{n j}\right\|_{\Gamma}=0$. Similarly,

$$
\begin{aligned}
\lim _{n} \sum_{j=1}^{n} \int_{\|x\|_{\Gamma} \leq \epsilon}\left(x-x_{n j}, y\right)^{2} d F_{n j}(x) & =\lim _{n} \int_{\|x\|_{\Gamma} \leq \sqrt{n} \epsilon}\left(x-x_{n j}, y\right)^{2} d \mu(x) \\
& =\int_{E_{a}}(x, y)^{2} d \mu(x)=T_{0}(y, y) .
\end{aligned}
$$


Finally (9.10) holds since $\sup _{n} \int_{\mathcal{U}} x_{i}^{2} d F_{n}(x)=\int_{E_{\alpha}} x_{i}^{2} d \mu(x)$.

We now derive some recent results on the central limit theorem due to A.D.de Acosta [121. A Banach space $X$ with a Schauder basis $\left\{b_{i}\right\}$ and coordinate functionals $\left\{\beta_{j}\right\}$ is said to be of type $p$ with respect to $\left\{b_{i}\right\}$ if

$$
P_{n}\left[\sum_{j=1}^{m} \beta_{j}(x) b_{j}\right]^{p} \leq \sum_{j=n+1}^{m}\left|\beta_{j}(x)\right|^{p}
$$

for all $x \in X$ and integers $m, n(1 \leq n \leq m)$ where, for each $n, x \in X$,

$$
P_{n}(x)=\inf \left\{\|x-z\|: z \in S_{n}\right\}
$$

with $S_{n}$ being the subspace generated by $\left\{b_{1}, \cdots, b_{n}\right\}$ for $n \geq 1$ and $S_{0}=0$.

Lemma 9.1. Let $X$ be of the type $p \geq 2$. Then there exists a linear isomorph $U$ on a subset $X_{0}$ of $X$ onto $l_{2}$ such that $\|U x\|_{l_{2}} \geq\|x\|_{X}$.

Proof. Since $X$ is of type $p \geq 2$ it is of type 2 [12]. Let us consider $X_{0}=$ $\left\{x: x \in X, \sum_{j=1}^{\infty}\left|\beta_{j}(x)\right|^{2}<\infty\right\}$. Define $U$ on $X_{0}$ to $l_{2}$ by $U x=\left(\beta_{1}(x), \beta_{2}(x)\right.$, ...). Then $U$ is an isomorph of $X_{0}$ onto $l_{2}$ and taking $n=0$ we have by (9.11) that $\|U x\|_{l_{2}} \geq\|x\|_{X}$.

Now let $T$ be a bilinear form defined on $X^{*}$. Then $T$ is said to be of trace class $p$ relative to $\left\{\beta_{j}\right\}$ if $\sum_{j=1}^{\infty}\left|T\left(\beta_{j}, \beta_{j}\right)\right|^{p}<\infty$.

We now state a lemma which strengthens the assertion of Theorem 4.1 (b) of $[12]$.

Lemma 9.2. Let $X$ be a Banach space with Schauder basis of type $p \geq 2$. Let $\left\{\mu_{\alpha}: \alpha \in A\right\}$ be a set of probability measures on $X$. Suppose for every $\epsilon>0$, there exists a family $\left\{T_{a, \epsilon}\right\}$ of nonnegative bilinear forms of trace class one satisfying

$$
\begin{gathered}
\sup _{\alpha \in A} \sum_{j=1}^{\infty} T_{a, \epsilon}\left(\beta_{j}, \beta_{j}\right)<\infty, \\
\lim _{N} \sup _{a \in A} \sum_{j=N}^{\infty} T_{a, \epsilon}\left(\beta_{j}, \beta_{j}\right)=0, \\
\left|1-\operatorname{Re} \phi\left(y, \mu_{\alpha}\right)\right| \leq T_{a, \epsilon}(y, y)+\epsilon \quad\left(y \in X^{*}\right) .
\end{gathered}
$$

Then $\left\{\mu_{a}: a \in A\right\}$ is supported on $X_{0}$ and it is conditionally compact on $X_{0}$ where the norm $\|x\|_{X_{0}}=\|U x\|_{l_{2}}$.

Proof. Apply Theorem 2.2 of [3]. 
In view of Lemma 9.2 we can prove the central limit theorem of [12] with $p \geq 2$. That is, under the hypothesis of the theorem and by Lemma 9.2 the measures $\Pi_{j=1}^{k} F_{n j}$ are conditionally compact on $X_{0}$. Further, the classical central limit theorem says the limiting measure must be Gaussian with Fourier transform as indicated. We also point out, but we will not provide the computations, that the hypotheses on the central limit theorem of [12] with $p \geq 2$ actually imply the hypotheses of Theorem.9.2 in case the measures sit on $l_{2}$. This, however, in view of Lemma 9.2 , is always the case under such conditions.

\section{BIBLIOGRAPHY}

1. N. Dunford and J.T. Schwartz, Linear operators. I: General theory, Pure and Appl. Math., vol. 7, Interscience, New York, 1958. MR 22 \#8302.

2. L. Gross, Harmonic analysis on Hilbert space, Mem. Amer. Math. Soc. No. 46 (1963). MR $28 \# 4304$.

3. J. Kuelbs and V. Mandrekar, Harmonic analysis on certain vector spaces, Trans. Amer. Math. Soc. 149 (1970), 213-231.

4. J.L. Kelley, General topology, Van Nostrand, Princeton, N.J., 1955. MR 16, 1136.

5. Ju. V. Prohorov, Convergence of random processes and limit theorems in probability theory, Teor. Verojatnost. i Primenen. 1 (1956), 177-238= Theor. Probability Appl. 1 (1956), 157-214. MR 18, 943.

6. Ju. V. Prohorov and V.V. Sazonov, Some results related to Bochner's theorem, Teor. Verojatnost. i Primenen. 6 (1961), 87-93 = Theor. Probability Appl. 6 (1961), 8286. MR $26 \# 6729$.

7. V.V. Sazonov, On characteristic functionals, Teor. Verojatnost. i Primenen. 3 (1958), 201-205 = Theor. Probability Appl. 3 (1958), 201 -205. MR 20 \#4882.

8. N.N. Vakhania, Sur une propriété des répartitions normales de probabilités dans les espaces $l_{p}(1 \leq p<\infty)$ et $H$, C.R. Acad. Sci. Paris 260 (1965), 1334-1336. MR 30 \#4282.

9. - Sur les répartitions de probabilités dans les espaces de suites numériques, C.R. Acad. Sci. Paris 260 (1965), 1560-1562. MR 30 \#4283.

10. A.C. Zaanen, Linear analysis, measure and integral, Banach and Hilbert space, linear integral equations, Interscience, New York; North-Holland, Amsterdam; Noordhoff, Groningen, 1953. MR 15, 878.

11. S.R.S. Varadhan, Limit theorems for sums of independent random variables with values in a Hilbert space, Sankhyā Ser. A 24 (1962), 213-238. MR 30 \#1536.

12. A.D. de Acosta, Existence and convergence of probability measures in Banach spaces, Trans. Amer. Math. Soc. 152 (1970), 273-298. MR $42 \# 2516$.

13. L. LeCam, Remarques sur le théorème limite central dans les espaces localement convexes, Les Problèmes sur les Structures Algébriques, Colloq. de la Recherche Scientifique, Paris, 1970, pp. 233-249.

DEPARTMENT OF MATHEMATICS, UNIVERSITY OF WISCONSIN, MADISON, WISCONSIN 53706

DEPARTMENT OF STATISTICS AND PROBABILITY, MICHIGAN STATE UNIVERSITY, EAST LANSING, MICHIGAN 48823 